\title{
SYNERGISTIC APPROACH OF ASTEROID EXPLOITATION AND PLANETARY PROTECTION
}

\author{
J.P. Sanchez \\ Advanced Space Concepts Laboratory, Department of Mechanical and Aerospace engineering, University of \\ Strathclyde, James Weir Building, 75 Montrose Street G1 1XJ, UK. Tel:(+44)1415485752 \\ email:jpau.sanchez@strath.ac.uk \\ C.R. McInnes
}

Advanced Space Concepts Laboratory, Department of Mechanical and Aerospace engineering, University of Strathclyde, James Weir Building, 75 Montrose Street G1 1XJ, UK. Tel:(+44)1415482049

email:colin.mcinnes@strath.ac.uk

The asteroid and cometary impact hazard has long been recognised as an important issue requiring risk assessment and contingency planning. At the same time asteroids have also been acknowledged as possible sources of raw materials for future large-scale space engineering ventures. This paper explores possible synergies between these two apparently opposed views; planetary protection and space resource exploitation. In particular, the paper assumes a 5 tonne low-thrust spacecraft as a baseline for asteroid deflection and capture (or resource transport) missions. The system is assumed to land on the asteroid and provide a continuous thrust able to modify the orbit of the asteroid according to the mission objective. The paper analyses the capability of such a near-term system to provide both planetary protection and asteroid resources to Earth. Results show that a 5 tonne spacecraft could provide a high level of protection for modest impact hazards: airburst and local damage events (caused by 15 to 170 meter diameter objects). At the same time, the same spacecraft could also be used to transport to bound Earth orbits significant quantities of material through judicious use of orbital dynamics and passively safe aero-capture manoeuvres or low energy ballistic capture. As will be shown, a 5 tonne low-thrust spacecraft could potentially transport between 12 and 350 times its own mass of asteroid resources by means of ballistic capture or aero-capture trajectories that pose very low dynamical pressures on the object.

Keywords: Near-Earth Objects; Near-Earth Asteroids; Asteroid Deflection; Asteroid Exploitation; Space Utilization.

\section{INTRODUCTION}

The threat that asteroids pose to life on Earth has long been acknowledged (Kleiman, 1968). Initial concerns focused on the potential threat from mass extinction events, similar to that of the cretaceous-tertiary period (Alvarez et al. , 1980). These events, albeit having an almost vanishingly low probability of occurrence, could potentially have catastrophic consequences. First surveying efforts therefore focused on discovering all objects larger than $1 \mathrm{~km}$ diameter (Morrison, 1992), thus those with potential to cause global damage. After almost 20 years of asteroid surveys, the census of asteroids larger than $1 \mathrm{~km}$ diameter is almost complete and thus the remaining impact risk has gradually switched towards smaller objects.

A detailed analysis on impact hazard, carried out by (Stokes et al. , 2003), showed an important residual risk for objects smaller than $1 \mathrm{~km}$ diameter, with a maxima at objects of order 300 meters diameter. The latest analysis on impact risk (Shapiro et al., 2010), though, seem to refute (Stokes, Yeomans, 2003), drawing two clear risk bands: a residual global threat posed by plausible large undiscovered objects and a local threat posed by the undiscovered population of numerous small objects ranging from 10 meters to 100 meters diameter (see figure 2.7 in 2010 impact hazard report from the National Academies Press (Shapiro et al., 2010)). The reason for this recent change is two-fold: firstly, the latest estimates on the population of Near-Earth Objects (NEOs) show a drop on the expected number of objects between 10 to 500 meters diameter (Harris, 2007), with a maximum difference of a factor of 2 to 3 on the total accumulative number of asteroids of order 100 meters 
diameter; secondly, the capability to yield ground damage of the Tunguska type of events (Steel, 2008) seem to have been underestimated (Shapiro et al., 2010).

Work on asteroid deflection systems, for planetary protection purposes, has generally focused either on investigating the feasibility of new exotic methods, such as gravitational tractors (Edward and Stanley, 2005) or solar ablation systems (Vasile and Maddock, 2010) and many others, or on comparing different methods in terms of efficiency (some examples are (Ivashkin and Smirnov, 1995), (Sanchez et al. , 2009) and (PA\&E, 2007)). Some space agencies, such as NASA and ESA, have also carried out some detailed studies on possible deflection precursor missions (e.g., ESA's Don Quixote* mission).This paper, on the other hand, will focus on the possible synergy between space systems capable of deflecting realistic current impact threats and, at the same time, capturing small asteroids for later resource exploitation in the Earth's neighbourhood. The concept of capturing near Earth asteroids may prime future large-scale space engineering ventures such as space solar power, space tourism and human space exploration. A small captured object could also be a future science goal in order to allow near real-time telecommunications with landers. Importantly, as will be shown, these potentially capturable targets are sufficiently small that they pose no direct impact hazard to the Earth during the capture manoeuvres.

In particular, this work analyses a low-thrust tugboat as a system capable of both asteroid deflection and capture. The tugboat concept assumes a low-thrust propulsion spacecraft attaching itself to the surface of the asteroid and providing a continuous thrust. For the deflection scenario, the purpose of the thrust is clearly to move the asteroid away from a potential collision trajectory with Earth. The paper then first presents an assessment on the capability of such a system to deflect realistic impact threats.

The assessment assumes a 5 tonne payload launched to Earth escape with a rendezvous trajectory towards a virtual threatening asteroid and attempting to deflect it from its collision trajectory. This deflection process is then repeated over a set of more than 17 thousand virtual threatening asteroids, which have been carefully chosen to provide a comprehensive overview of all possible asteroid impact scenarios. The results from the set of deflection attempts provide a good estimate of the level of planetary protection that such a system could be capable of.

Secondly, the paper focuses on estimating the statistical population of small asteroids (i.e., diameters on the order of meters to tens of meters) that could potentially be manoeuvred and captured into a bound Earth orbit using the same tugboat system. This statistical population of capturable asteroids can be estimated by comparing the regions of accessible Keplerian orbital element space with a NEO model able to predict the statistical probability of the existence of an asteroid with a given set of both orbital elements and diameters. For the latter purpose the theoretical Near-Earth Object model published by Bottke et al. (Bottke et al. , 2002) will be used to estimate the orbital element distribution, whereas the size population estimates will consider Harris (Harris, 2007).

Since the only propulsion system used is low thrust, the asteroid orbital insertion at Earth must be provided either ballistically (i.e., a natural low energy gravitational capture into an Earth bound orbit) or by means of aero-assisted trajectories. The paper will show that by using very small aerobraking manoeuvres, posing dynamical pressures much smaller than those for which the object would be expected to fragment, a nonnegligible statistical population of asteroids can be captured within the Earth's gravity well. The low-thrust tugboat is here assumed to be used only to trim the orbital phasing of the object so that it meets the Earth at the orbital intersection, hence only objects with very well defined orbital geometries can be suitable targets for capture.

\section{TUGBOAT SYSTEM}

The baseline design of the tugboat system is fixed throughout the paper since the purpose of the paper is to investigate the deflection capability of a plausible deflection system, and then compare it with its capability to transport material to the Earth's neighbourhood. By plausible we mean here a mission of a similar size to current

*http://www.esa.int/SPECIALS/NEO/SEMZRZNVGJE_0.html 
missions to asteroids, for example NASA's Dawn mission (Rayman et al. , 2006) with a wet mass at launch of $1,250 \mathrm{~kg}$ or ESA's Rosetta mission ${ }^{\dagger}$ with a wet mass of 3,000 kg. It is envisaged then that a wet mass of 5,000 $\mathrm{kg}$ with an Earth escape velocity $v_{\infty}$ of $2.5 \mathrm{~km} / \mathrm{s}$ is a perfectly plausible mission requiring levels of funding comparable to ESA's cornerstone or NASA's flagship missions (i.e., large current science exploration missions). Thus, such a mission would still require a dedicated launch with a heavy lift launch vehicle, but the wet mass and excess velocity is within the current launch capabilities of Ariane V and Delta IV-H (PA\&E, 2007). Note that this is a relatively small mission if compared with both ambitious solar system exploration concepts $(>10,000 \mathrm{~kg}$ wet mass (Langmaier et al. , 2008)) and deflection systems for challenging impact scenarios $(>100,000 \mathrm{~kg}$ for cases with short warning times and/or large threatening objects (Sanchez, 2009)).

\subsection{The Spacecraft and Transfer}

Together with the concept of kinetic impactor (i.e., involving changing the asteroid's linear momentum by impacting a spacecraft onto it), the tugboat system is one of the simplest deflection concepts proposed for the purpose of impact hazard mitigation. The concept consists of a spacecraft landing on the asteroid and using its propulsion system to push the asteroid away from the impact trajectory. The preferred choice for the propulsion system is to use low thrust systems, since these provide specific impulses 10 times higher than chemical propulsion and therefore require significantly less reaction mass to impart the same change in momentum.

The drawback of the higher specific impulse is that its lower level of thrust requires a long duration to provide a useful change to the asteroid's trajectory. Due to this, the rotation of the asteroid becomes an issue, since on a rotating asteroid the thrust vector of the system will not maintain a constant pointing direction. The propulsion system will therefore have to be switched on and off when the correct thrusting direction occurs or, alternatively, the asteroid rotation will have to be modified so that the propulsion system can be continuously active (Scheeres and Schweickart, 2004). The first option, switching the propulsion system on and off when the pointing is within tolerable misalignments, is deemed to be much simpler to implement, since, for example, the asteroid-to-spacecraft attachment will not require a gimbaling system, while still providing adequate efficiency (Sanchez, Colombo, 2009).

The tugboat deflection system modelled in this paper assumes two equal spacecraft, each requiring half of the $5,000 \mathrm{~kg}$ launch mass, landing on opposite sides of the asteroid and thrusting through the centre of mass of the object. By properly scheduling the periods when the engines are switched on and off, we can obtain a quasiconstant thrust and a limited scattering factor. The scattering factor takes into account the misalignment from the optimal thrust direction, and by multiplying it by the thrust results in the effective force applied in the optimal thrusting direction. As shown in Sanchez et al. (Sanchez, Colombo, 2009), no matter the shape and obliquity of the asteroid's equator, it is always possible to achieve at least $25 \%$ of thrust efficiency, and mean efficiencies of $30 \%$, without changing the rotational state of the asteroid.

The mass of the system at the arrival at the asteroid $m_{i}$ includes the propellant mass for the deflection manoeuvre and the dry mass $m_{d}$ of the spacecraft. The dry mass $m_{d}$ defines the size of the power system $m_{\text {power }}$, which is assumed here to be $50 \%$ of the dry mass of the spacecraft (this includes common payload mass fractions into the power subsystem (James and Wiley, 2003)). The thrust capability $T$ of the system can then be estimated using the following linear relationship:

$$
T=m_{\text {power }} \frac{\xi}{\tau}
$$

where the specific thrust $\xi$ is set equal to $34 \mathrm{mN} / \mathrm{kW}$, which represents an average value for the most common ion thrusters (James and Wiley, 2003), and the mass-to-power ratio $\tau$ is set to $25 \mathrm{~kg} / \mathrm{kW}$ as an average for the power systems most commonly used in space (see table 11-33 in (James and Wiley, 2003)). Note that $T$ is the total thrust of the system and that each half-spacecraft landing on opposite sides of the asteroid will have a thrust $T / 2$. The proportion of the mass at arrival $m_{i}$ which is dry mass $m_{d}$ or propellant for thrusting also requires to be optimized to achieve the maximum deflection. This proportion is defined by the length of the thrusting operation and is not necessarily the same as the time left to impact, since shorter push manoeuvres allow the transfer of

\footnotetext{
${ }^{\dagger} \mathrm{http} / /$ sci.esa.int/science-e/www/area/index.cfm? fareaid=13
} 
higher levels of impulse to the asteroid earlier, which then enhances the deflection achieved by the passive drift of the asteroid relative to the Earth. Further details on the system can be found in (Sanchez, 2009, Sanchez, Colombo, 2009).

In order to compute the mass at arrival $m_{i}$, the cost of the rendezvous trajectory with the asteroid needs to be estimated. The propulsion system used is a low-thrust system, and therefore, the trajectory should ideally be computed using a low-thrust optimization technique such as, for example, optimal control theory (Betts, 1998) in order to optimize thrust direction and thrust duration. Unfortunately, the work presented here required the optimization of the trajectory to over 17 thousand asteroids, and therefore, a full low thrust optimization would have taken an unfeasibly long computational time. Even a first guess method for low thrust, such a shape-based approach (De Pascale and Vasile, 2006), would have required orders of magnitude longer than a simple Lambert-arc optimization. Thus, trajectories have been computed by optimizing the minimum $\Delta v$ Lambert-arc connecting the asteroid and Earth's orbits and considering the first $2.5 \mathrm{~km} / \mathrm{s}$ at departure as provided by the launcher (this simple procedure required 4 weeks of computational time on a Intel Core 2 at $3 \mathrm{GHz}$ ). This, of course is only a good approximation if the transfer is impulsive, and thus, a gravity loss penalty due to the limited thrust needs to be accounted for. Figure 1 shows the numerically computed penalty for thrusting from a circular $1 \mathrm{AU}$ orbit to escape velocity as a function of the available thrust and considering the 5,000 $\mathrm{kg}$ initial mass and an $I_{\mathrm{sp}}$ of $3000 \mathrm{~s}$. The penalty is computed by comparing the integrated velocity change $\Delta v$ of the limited thrust escape with the theoretical impulsive escape velocity. As can be seen in the figure, a factor of 2.1 times the $\Delta v$ computed by means of a minimum Lambert-arc should approximately compensate for the limited thrust of the tugboat.

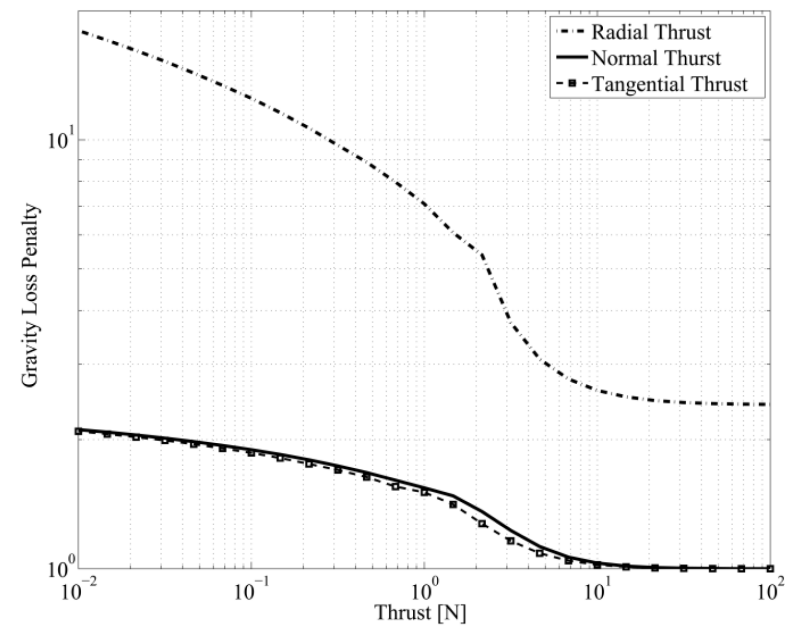

Figure 1: Gravity loss penalty for a 5-tonne spacecraft continuously thrusting to escape velocity from an initial circular $1 \mathrm{AU}$ orbit.

At the arrival of the tugboat at the asteroid the mass of the system can be reduced according the $2.1 \times \Delta v$ cost necessary for rendezvous with the specific asteroid. The final mass at arrival $m_{i}$ can be computed by means of Tsiolkovsky's rocket equation and assuming an $I_{\mathrm{sp}}$ of 3000 seconds, as a mean specific impulse of common ion thrusters (James and Wiley, 2003). Figure 2 shows the tugboat mass at arrival $m_{i}$ as function of the $\{a, e, i\}$ position of the virtual impactor at which the tugboat is set to rendezvous. 


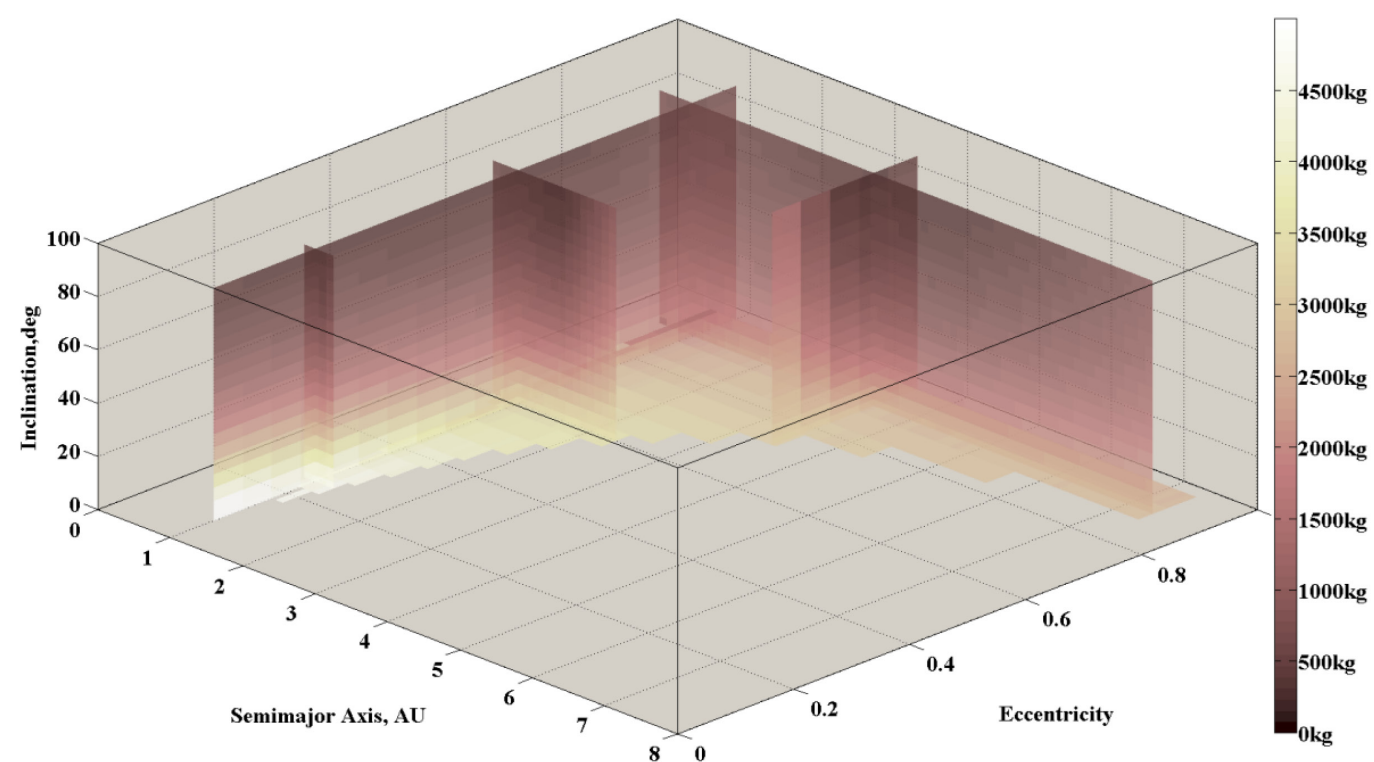

Figure 2: Tugboat mass at arrival $m_{i}$ at the virtual impactor. The set of solutions $m_{i}(a, e, i)$ are represented through a set of representative slice planes on the 3D matrix of solutions.

We refer here to a lead time as the time spanned from the moment at which the tugboat system is deployed and ready at the asteroid until the collision time. Within the available lead time, a push maneuvre will be optimized for each asteroid in order to achieve a maximum deflection distance. As noted earlier and as shown in previous work (Sanchez, Colombo, 2009), the tugboat system requires long duration push manoeuvres, i.e., several years, in order to achieve acceptable deflection distances. The lead time then is a crucial parameter of the deflection scenario. Here, five representative lead times have been chosen for analysis; 1, 2.5, 5, 10 and 20 years. This small set of lead times offers the compromise of providing an assessment of the influence of the lead time on planetary protection, while avoiding a huge increase in the computational time required. Note that for each different lead time used, more than 17 thousand optimizations need to be run to compute the deflection for each of the asteroids in the set.

Finally, the warning time, defined as the total time spanned from launch to the collision time, may be substantially longer than the lead time, since transfers to some of the asteroids are long. In fact, the average warning time for the set of lead times chosen is $9,11,13,17$ and 27 years respectively to the set of lead times $(1,2.5,5,10$ and 20 years). Note that this is considering perfect launch opportunities (i.e., free phasing in the Lambert-arc) and accounting for phasing problems would certainly increase the mean warning time. However, even if these warning times appear to be long, we can assume by extrapolating current asteroid survey efforts that future impact threats may be detected decades prior to collision.

\section{DEFLECTION SCENARIO}

As noted previously, over 17 thousand virtual impactors were created in order to provide a comprehensive overview of asteroid impact scenarios. The set of virtual impactors is built by defining a set of homogeneously distributed impact trajectories, as will be now described. The largest asteroid mass that the 5-tonne tugboat (see Section 2) is capable of deflecting to a safe distance from Earth can be computed for each one of the 17 thousand impacting trajectories, and with it the level of planetary protection that can be expected will be defined.

In order to build the set of impactors, the $\{a, e, i\}$-domain was first divided into a three-dimensional grid containing over 28,000 points homogeneously distributed within a semimajor axis $a$ from 0.05 to $7.35 \mathrm{AU}$, eccentricity $e$ from 0.025 to 0.975 and inclination $i$ from 0 to 87.5 degrees. Only 8,759 locations in this grid correspond to orbits with a perihelion smaller and aphelion larger than $1 \mathrm{AU}$ (see grid points in Figure 3), which is a necessary condition, but not sufficient, for an impact trajectory. Hence, only these positions in the grid allow 
a potential geometry such that the Minimum Orbital Intersection Distance, or MOID, (i.e., minimum possible distance between the Earth and the asteroid considering free-phasing for both objects) is zero (or close to).

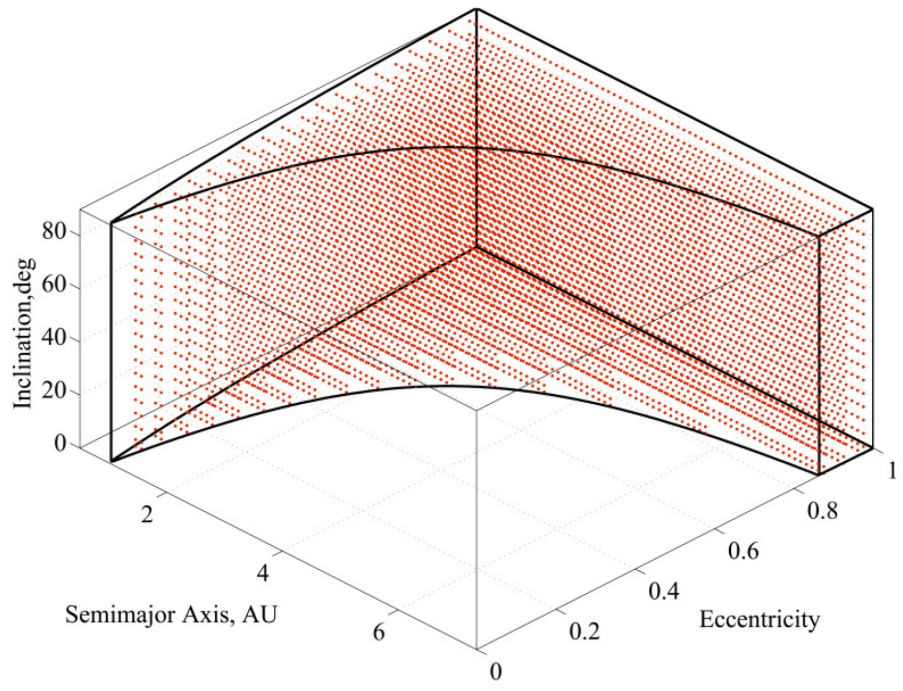

Figure 3: Grid used to create the set of virtual impactors.

Without loss of generality, the paper has assumed a circular 1 AU orbit as the orbit of the Earth. Under this approximation, and for each point in the grid represented in Figure 3, one can easily compute the ascending node $\Omega_{\text {impact }}$ and the argument of periapsis $\omega_{\text {impact }}$ that allows an object with a fixed semi-major axis $a$, eccentricity $e$ and inclination $i$ to cross the orbit of the Earth at a given angular position. As is shown in Figure 4, $\Omega_{\text {impact }}$ is uniquely defined by the position of the Earth at the fixed epoch at which the virtual impact is set (denoted by cross in the figure), while $\omega_{\text {impact }}$ has two possible configurations (note that $\omega_{\text {impact }}$, in Figure 4 , corresponds to the argument of the orbit with apoapsis below the orbit of the Earth, while the opposite orbit would be represented by $\left.-\omega_{\text {impact }}\right)$. Hence, the points in the grid represented by Figure 3 define 17,518 impact trajectories, referred here as virtual impactors, that have been used to analyse the deflection capability of the tugboat defined in Section 2.

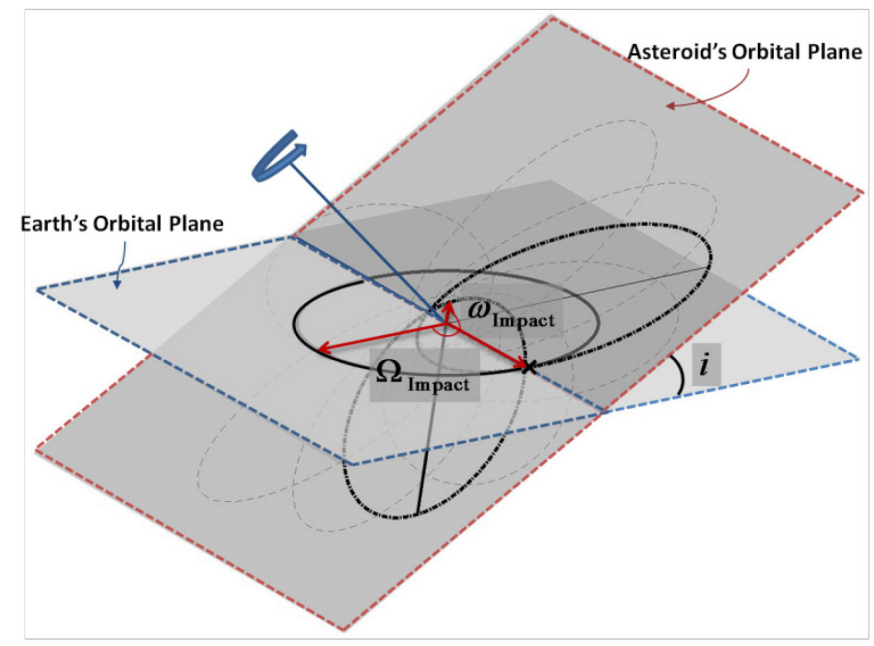

Figure 4: Orbital geometry of possible impactors for a given semi-major axis $a$, eccentricity $e$ and inclination $i$.

As mentioned, each virtual impactor from the set is defined by the set of Keplerian elements leading to an impact at a given epoch. Note then that the impact velocity of each asteroid is now also defined, since the orbits of both objects Earth and asteroid are known. The impact energy, on the other hand, requires knowing the mass of the impacting object. The objective then is to compute the mass of the largest object that the tugboat system can deflect from each one of the impacting trajectories in order to understand the deflection capability of the system. 


\subsection{Impact Probability}

Each of the virtual impactors represented in Figure 3 does not have the same likelihood to exist. There are regions of the Keplerian element space much more densely populated than other regions. For example, there are many more low inclination, than high inclination, asteroids. On the other hand, there are regions that allow more or better opportunities to impact the Earth. Therefore, the relative Earth impact frequency of each impactor in Figure 3 needs to be assessed individually, by means of two multiplying factors: first, the NEA orbital distribution that defines the actual asteroid probability density; and second, the collision probability of a given set of $\{a, e, i\}$, which assesses the likelihood of impact of a given object.

\section{NEO Orbital Distribution}

The NEO orbital distribution used here is based on an interpolation from the theoretical distribution model published in Bottke et al. (Bottke, Morbidelli, 2002). The data used was very kindly provided by W.F. Bottke (personal communication, 2009). Bottke et al. (Bottke, Morbidelli, 2002) built an orbital distribution of NEOs by propagating in time thousands of test bodies initially located at all the main source regions of asteroids (i.e., the $v 6$ resonance, intermediate source Mars-crossers, the 3:1 resonance, the outer main belt, and the transNeptunian disk). By using the set of asteroids discovered by Spacewatch at that time, the relative importance of the different asteroid (or comets) sources could be best-fitted. This procedure yielded a steady state population of near Earth objects from which an orbital distribution as a function of semi-major axis $a$, eccentricity $e$ and inclination $i$ can be interpolated numerically. Figure 5 shows a representation of Bottke's NEA density $\rho(a, e, i)$.

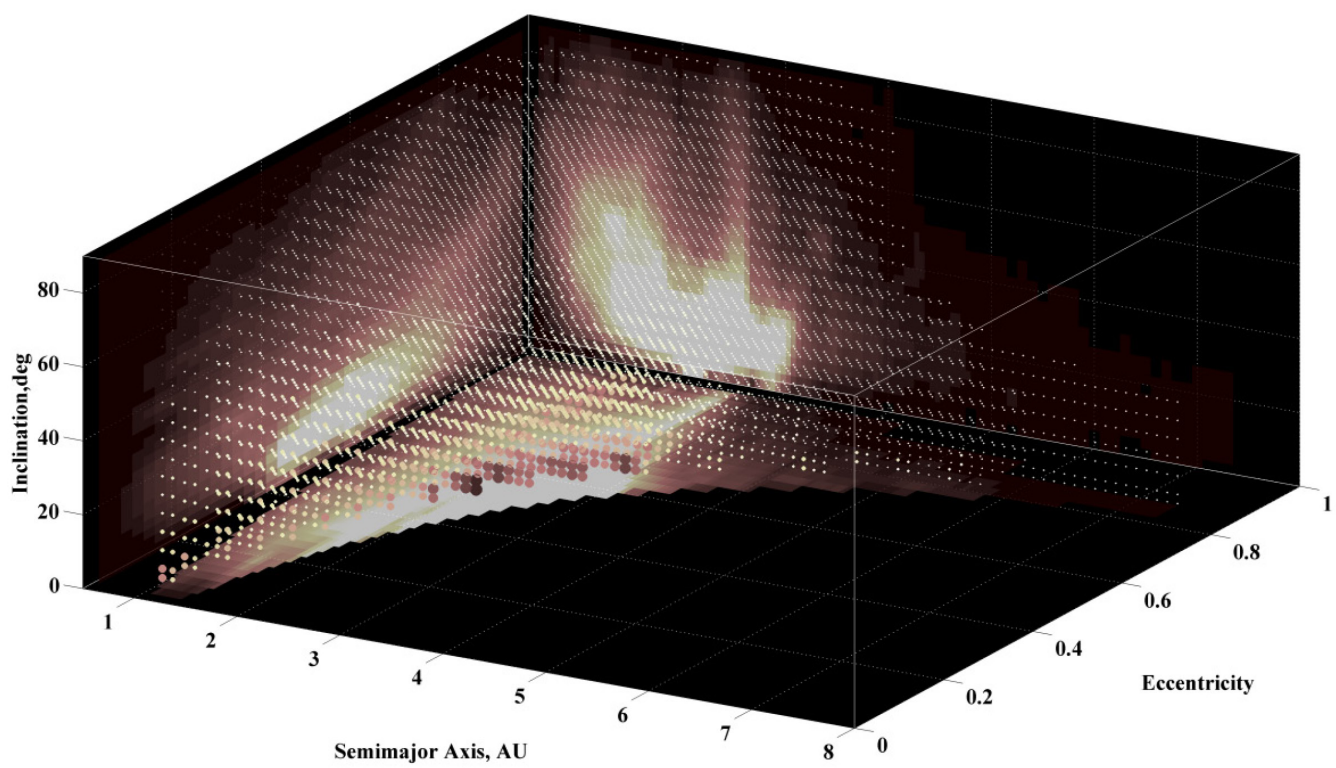

Figure 5: Theoretical Bottke et al. (Bottke, Morbidelli, 2002) NEA distribution. The figure shows the integrated projections of function $\rho(a, e, i)$ and a set of grid points coloured and sized linearly with the values of the NEO density.

\section{Collision Probability of an Asteroid}

A necessary condition, for an asteroid to impact the Earth, is to have both a perihelion smaller and aphelion larger than $1 \mathrm{AU}$. This, of course, is not a sufficient condition, since only a very limited set of arguments of periapsis $\omega$ will actually yield a trajectory crossing Earth's orbital path (see dashed trajectories in the asteroid's orbital plane in Figure 4). For a given epoch, hence a fixed position of the Earth within its orbital path, only a single ascending node $\Omega$ allows the asteroid trajectory to intersect the Earth (see again Figure 4). Finally, of course, only one possible mean anomaly $M$ allows the asteroid to meet the Earth at the same exact position. Hence, the NEO density distribution $\rho(a, e, i)$, which defines the probability of finding an asteroid (or comet) with given $\{a, e, i\}$ based on known asteroid population evolution, is not yet a measure of how likely is to find an impactor with a given set of Keplerian elements. 
The present section will now define the collision probability function $g(a, e, i)$ which defines the relative frequency which an asteroid with a given $\{a, e, i\}$ should meet the Earth along its trajectory. As previously defined, the MOID is the minimum orbital distance possible between two orbits and, particularly, in the case at hand between the Earth and an asteroid. In order to compute the collision probability of an asteroid with Keplerian elements $\{a, e, i\}$, we first need to compute the maximum MOID that allows an Earth collision. For the latter, the Earth's gravity needs to be accounted for, since an asteroid close to Earth will essentially follow a hyperbolic trajectory with the Earth at its focus. A hyperbolic factor $\varepsilon$,

$$
\varepsilon=\frac{r_{a}}{r_{p}}=\sqrt{1+\frac{2 \mu_{\oplus}}{r_{p} v_{\infty}^{2}}}
$$

accounts then for the curvature that the object's trajectory would experience during the Earth approach. In Eq.(2), $r_{\mathrm{a}}$ is the minimum distance between the hyperbola asymptote and the Earth, $r_{\mathrm{p}}$ is perigee distance of the asteroid's hyperbolic trajectory, $\mu_{\oplus}$ is the gravitational constant of the Earth and $v_{\infty}$ the hyperbolic excess velocity of the asteroid. Thus, if we assume that the maximum distance for a collision to occur is one Earth radius $r_{\oplus}$, the actual maximum geometrical distance between the orbit of the Earth and the asteroid will require to be smaller than,

$$
\mathrm{MOID}_{\oplus}=r_{\oplus} \sqrt{1+\frac{2 \mu_{\oplus}}{r_{\oplus} \cdot v_{\infty}{ }^{2}}} .
$$

Note that $M O I D_{\oplus}$ is a function of the three Keplerian elements $\{a, e, i\}$, since the hyperbolic velocity $v_{\infty}$ can be described as:

$$
v_{\infty}=\sqrt{\mu_{s}\left(3-1 / a-2 \sqrt{a\left(1-e^{2}\right)} \cdot \cos (i)\right)}
$$

where $\mu_{S}$ is the gravitational constant of the Sun and both semi-major axis $a$ and $\mu_{S}$ need to be expressed in AU units, thus units of length in $v_{\infty}$ are also AU. Equation (4) can be derived from Tisserand's parameter or, alternatively, from the relative velocity components of the asteroid (at a distance $1 \mathrm{AU}$ from the Sun) and the Earth circular motion (Opik, 1951). These are described later in the text and computing its modulus one reaches the expression in Eq.(4).

The section will proceed by defining an analytical approximation of the MOID that allows to easily compute the range of argument of the periapsis $\omega$ such that the asteroid's MOID is smaller than $M O I D_{\oplus}$. Two important assumptions allow us to proceed: firstly, we have already assumed a circular $1 \mathrm{AU}$ orbit for the motion of the Earth, as for Eq.(4), and secondly, we assume that the right ascension of the ascending node $\Omega$ and the argument of periapsis $\omega$ are uniformly distributed random variables. The latter assumption will allow us to estimate the probability of an asteroid to have an argument of the periapsis $\omega$ such that its MOID is smaller than $M O I D_{\oplus}$. The ascending node $\Omega$ and the argument of periapsis $\omega$ are generally believed to be uniformly distributed in near Earth orbit space as a consequence of the fact that the period of the secular evolution of these two angles is expected to be much shorter than the life-span of a near Earth object. Therefore, we can assume that any value of $\Omega$ and $\omega$ is equally possible for any NEO (Bottke, Morbidelli, 2002). Similarly, all values of mean anomaly $M$ are also assumed to be equally possible, and thus $M$ is uniformly distributed too.

\section{Minimum Orbital Intersection Distance:}

The exact values of $\omega_{\text {impact }}$ are relatively easy to find by noticing that the true anomaly at the ecliptic plane must be such that the distance to the Sun is 1AU. Two of these are possible values of $\omega_{\text {impact }}$ for an Earthcrossing asteroid to have a collision with the Earth (as can be seen in Figure 4). While an asteroid with argument of periapsis equal to $\omega_{\text {impact }}$ will have a MOID equal to zero, for argument of periapsis close to $\omega_{\text {impact }}$ the variation of MOID can be approximated linearly (Opik, 1951). With the axes shown as in Figure 6, the motion of the Earth and the asteroid can be described using a linear approximation of the Keplerian velocities of 
the two objects at the encounter. This defines two straight line trajectories, and thus, the minimum distance between these two linear trajectories can be found. The minimum distance can then be written as an explicit function of $\Delta x$, which is defined here as the distance between the centre of the coordinates described in Figure 6 and the point at which the asteroid crosses the Earth's orbital plane. This minimum distance $\Delta x$ can alternatively be described as a linear function of the argument of the periapsis $\omega$. Finally, an expression such as (Opik, 1951):

$$
\operatorname{MOID}(\omega)=\frac{\left|\omega_{\text {impact }}-\omega\right|}{\sqrt{\left(\frac{1}{\sin (i)}\right)^{2}+\tan ^{2}(\alpha)}}
$$

yields an approximate value of the MOID distance. The absolute value $\left|\omega_{\text {impact }}-\omega\right|$ refers to the minimum absolute difference between the two values of $\omega_{\text {impact }}$ while the tangent of the flight path $\alpha$ angle can be found from:

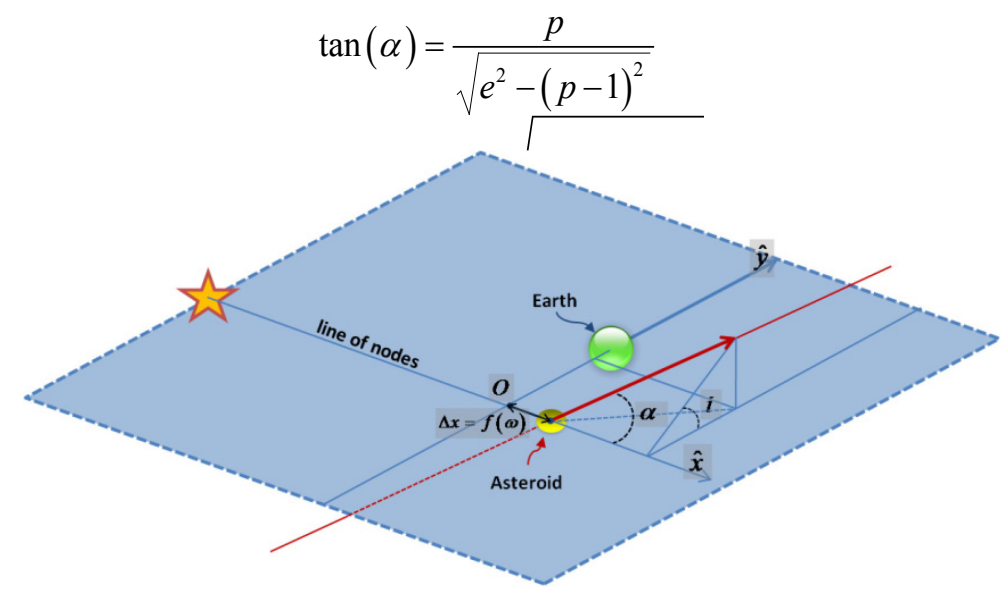

Figure 6: Set of coordinates used to compute Equation (5).

As can be seen in (Sanchez and McInnes, 2011a), Eq.(5) provides a good approximation of the numerically calculated MOID even for values not extremely close to $\omega_{\text {impact. }}$

Probability of having a MOID lower than the collision distance:

Since Eq.(3) defines the maximum MOID at which a collision would occur, rearranging Eq.(5) one can define the range of arguments of periapsis for which an asteroid would have a MOID smaller than $M O I D_{\oplus}$ :

$$
\Delta \omega=\operatorname{MOID}_{\oplus} \cdot \sqrt{\left(\frac{1}{\sin (i)}\right)^{2}+\tan ^{2}(\alpha)} .
$$

From Eq.(7), $2 \Delta \omega$ provides the total range of $\omega$ that yields a MOID smaller than $M O I D_{\oplus}$ for each impactor in Figure 4, and thus the total range of arguments shall be $4 \Delta \omega$. Lastly, since the argument $\omega$ has been assumed to be a uniformly distributed random variable and the total range of possible arguments $\omega$ is $2 \pi$, the probability of having an argument of periapsis $\omega$ such that the impact can occur is:

$$
g(a, e, i)_{\omega}=\frac{2 \cdot(2 \cdot \Delta \omega)}{2 \pi} .
$$

\section{Probability of collision:}

Equation (8) has defined the probability of having an asteroid such that MOID is small enough for a collision to be possible, nevertheless we still require to know the probability of also having the Earth and the asteroid with a phasing such that the collision occurs. This will now be described by defining the function $g(a, e, i)_{c o l}$ or probability of collision with Earth of an asteroid with MOID $\leq M O I D_{\oplus}$. 
As is well known, the shortest distance between two linear trajectories must have a direction perpendicular to both of them, and this must also be satisfied by the Earth and asteroid linear trajectories as described in Figure 6. As depicted now in Figure 7, this condition defines a cylindrical region around the Earth linear trajectory that must be crossed by any object with a minimum orbital distance smaller than the radius of the cylinder. Since the motion of an asteroid with defined inclination $i$ (and ascending node $\Omega$ ) will always be confined within its orbital plane, an elliptical section can be defined as a result of the intersection between the cylinder of radius $r$ and the asteroid's orbital plane (see Figure 7). Note that since the Earth is assumed to be on a circular orbit, the right ascension of the ascending node $\Omega$ is actually irrelevant for the calculation of the impact probability.

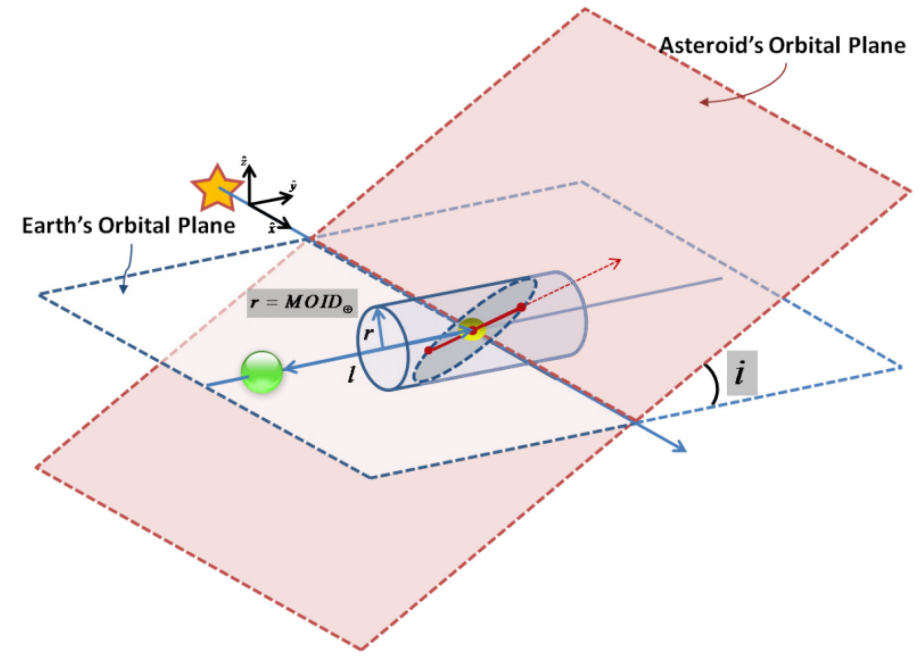

Figure 7: Representation of the Earth and asteroid impact geometry. Trajectories of both objects are assumed to be straight lines. An asteroid with a MOID smaller than $M O I D_{\oplus}$ requires to cross the elliptical intersection between the cylinder of radius $M O I D_{\oplus}$ and the asteroid's orbital plane.

Thus, as defined in Figure 7, a necessary, but not yet sufficient, condition for an asteroid to encounter the Earth at distance smaller than $r$ is that its trajectory must intersect the elliptical section drawn by the intersection of a cylinder of radius $r$ and the asteroid's orbital plane. For the case of a cylinder of radius equal to $M O I D_{\oplus}$, only asteroids with arguments of periapsis within $\omega_{\text {impact }} \pm \Delta \omega$ will have trajectories intersecting its elliptical section. Each of these trajectories (i.e., with varying $\omega$ within the range $\omega_{\text {impact }} \pm \Delta \omega$ ) will follow parallel paths, due to the linearly approximated motion, intersecting the elliptical section for a different length, as shown in Figure 8. Among all possible trajectories, the central passage yields the trajectory with the longest path within a distance smaller than $M O I D_{\oplus}$. The collision probability of each particular trajectory can then be assumed to be proportional to the path length within the elliptical section.

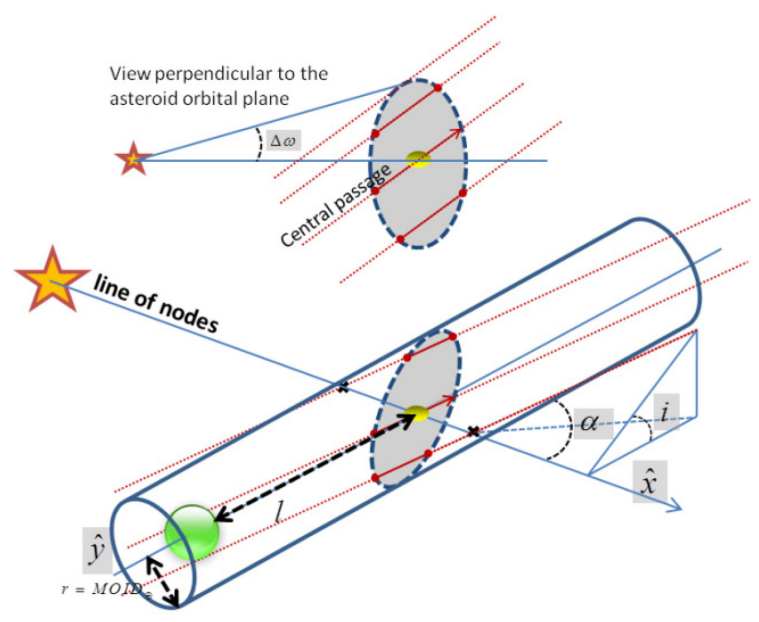

Figure 8: Configuration of asteroid impacting trajectories. All trajectories would draw parallel chords in the elliptical section. The ellipse's central trajectory correspond to the orbit with MOID equal to zero. 
These intersecting trajectories can also be seen as a set of parallel chords of the elliptical section. Then, the average length of the set of parallel chords (or parallel trajectories) crossing an ellipse can be computed to be $\pi / 4$ times the length of the central chord/trajectory (i.e., trajectory crossing the centre of the ellipse). Similarly then, the average probability of collision for asteroids with periapsis argument within the range $\omega_{\text {impact }} \pm \Delta \omega$ is assumed to be $\pi / 4$ times the probability of collision of the central trajectory:

$$
g(a, e, i)_{c o l}=\frac{\pi}{4} \cdot P(a, e, i)_{c o l: M O I D=0}
$$

where $P_{\text {col:MOID }=0}$ refers to the probability of collision with the Earth of the asteroid trajectory with MOID equal zero (MOID-zero object), or the central passage of the ellipse pictured in Figure 7 and Figure 8.

In order to compute $P_{c o l: M O I D}=0$, let us imagine the asteroid at the centre of the ellipse, or point of MOID equal to zero, while the Earth is at a distance $l$ from the same point (see Figure 8). The relative motion of these two bodies in radial-transversal-out-of-plane Cartesian coordinates and using AU as unit length is given by:

$$
\left(\sqrt{\frac{\mu_{s}}{p}\left(e^{2}-(p-1)^{2}\right)}, \sqrt{\mu_{s} p} \cdot \cos (i)-\omega_{\oplus}, \sqrt{\mu_{s} p} \cdot \sin (i)\right)
$$

where the Earth angular velocity $\omega_{\oplus}$ is equal to $\sqrt{\mu_{S}}$. Note also that Eq.(4) can be derived from Eq.(10). Using Eq.(10), the minimum distance between the two objects can be calculated and results in:

$$
d_{\min }=\sqrt{l^{2}\left(1-\frac{\mu_{S}(\sqrt{p} \cdot \cos (i)-1)^{2}}{v_{\infty}^{2}}\right)}
$$

If we then set the minimum distance $d_{\min }$ equal to the maximum MOID required for a collision, $M O I D_{\oplus}$, and isolate the variable $l$, we then obtain the range of positions for which the Earth would actually be impacted by a MOID-zero object at the MOID point:

$$
l_{\max }=\frac{M O I D_{\oplus} \cdot v_{\infty}}{\sqrt{\left(v_{\infty}^{2}-\mu_{S}(\sqrt{p} \cdot \cos (i)-1)^{2}\right)}} .
$$

Finally, $P_{\text {col:MOID }=0}$ can be easily computed by dividing the total length of possible Earth configurations allowing it to impact the MOID-zero asteroid, i.e., $2 l_{\max }$, by the total Earth path length in AU units, i.e., $2 \pi$.

Collecting the four previous subsections, the collision probability of an asteroid is then given by:

$$
g(a, e, i)=g_{\omega} \times g_{c o l}=\frac{l_{\max } \cdot \Delta \omega}{2 \pi} .
$$

Note that for very low inclinations or very small eccentricities, both $\Delta \omega$ and $l_{\max }$ tend to infinity, and thus, the linear approximation ceases to be valid. To avoid this problem the upper bound of $\Delta \omega$ is set to $\pi / 2$, while a numerical search of $l_{\max }$ is performed when the linearly approximated $l_{\max }$ becomes larger than 0.0175 radians (i.e., $1 \mathrm{deg}$ ). Similarly to the linear approximation, the numerical search finds the range of the Earth's mean anomaly $\Delta M_{\max }$ for which the minimum distance to a MOID-zero asteroid is equal to $M O I D_{\oplus}$.

\section{Relative Frequency of impactors}

The set of impactors can finally be weighted with their relative frequency in order to distinguish which regions of the Keplerian element space actually yield a higher impact risk. To compute the relative frequency, the impact probability $f_{I}(a, e, i)=\rho \cdot g$, where $\rho$ is the NEO density distribution and $g$ the collisional probability, is integrated along the $2 \Delta a \times 2 \Delta e \times 2 \Delta i$ box centred at each point of the grid represented in Figure 
3, where $\Delta a, \Delta e, \Delta i$ are the step sizes of the grid. Figure 8 shows again the grid represented in Figure 3, but this time each individual point in the grid is colored and sized accordingly to its relative frequency. As shown by Figure 8, there is a clear tendency for impactors with low inclination and Apollo-type orbits.

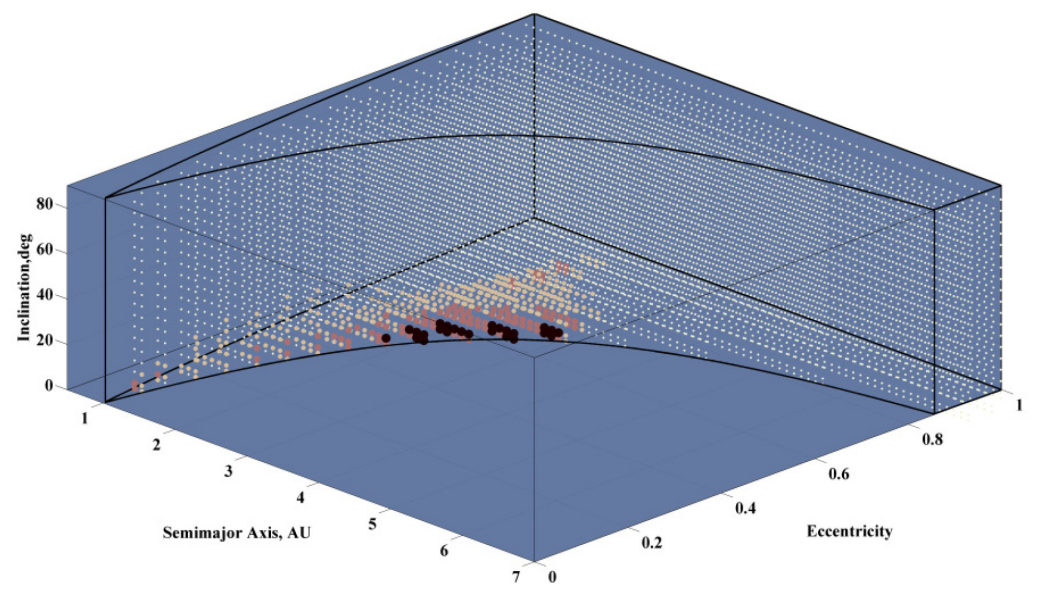

Figure 9: Set of virtual impactors plotted as dots of size and colour as a function of the relative frequency that should be expected for each impactor. Four different dot-types have been used in this figure to represent average relative impact probabilities as follow: $\bullet<\mathrm{P}>\approx 1 \%, \bullet<\mathrm{P}>\approx 0.3 \%$, $<\mathrm{P}>\approx 0.07 \%$ and the smallest refers to $<\mathrm{P}><0.01 \%$

\subsection{Expected planetary protection.}

The level of planetary protection that the tugboat is capable of providing can now be estimated. Recall that each virtual impactor has been defined as a Keplerian set of elements defining an Earth-impacting orbit. Since the Earth-impact energy can be estimated as:

$$
E_{\text {impact }}=\frac{1}{2} M_{\text {Ast }} v_{\text {impact }}^{2},
$$

where the impact velocity $v_{\text {impact }}$ of each asteroid is computed by:

$$
v_{\text {impact }}=\sqrt{\mu_{\oplus}\left(\frac{2}{r_{\oplus}}+\frac{v_{\infty}^{2}}{\mu_{\oplus}}\right)}
$$

and $v_{\infty}$ is computed as in Eq.(4), the maximum deflected impact energy $E_{\text {impact }}$ for each impacting orbit is the result of computing the maximum asteroid size $M_{A s t}$ that the tugboat is able to deflect with a given lead time. The energy of the impact event is also a more convenient way of categorising the different impact threats. By following, approximately, the definitions proposed in (Shapiro et al., 2010), the impact hazard can be divided into the six categories defined by their range of impact energy, as described in Table 1. Assuming also spherical asteroids with average density ( $2600 \mathrm{~g} / \mathrm{m}^{3}$ (Chesley et al. , 2002)), an approximate equivalent size range can be estimated for each impact level by considering the extreme variations of the impact velocity of the set of impactors considered in this paper.

Table 1: Impact hazard categories

\begin{tabular}{|c|c|c|}
\hline Type of Event & $\begin{array}{c}\text { Approximate range of Impact } \\
\text { Energies (MT) }\end{array}$ & $\begin{array}{c}\text { Approximate Range Size } \\
\text { of Impactor }\end{array}$ \\
\hline Airburst & 1 to $10 \mathrm{MT}$ & 15 to $75 \mathrm{~m}$ \\
\hline Local Scale & 10 to $100 \mathrm{MT}$ & 30 to $170 \mathrm{~m}$ \\
\hline Regional Scale & 100 to $1,000 \mathrm{MT}$ & 70 to $360 \mathrm{~m}$ \\
\hline Continental Scale & 1,000 MT to $20,000 \mathrm{MT}$ & $150 \mathrm{~m}$ to $1 \mathrm{~km}$ \\
\hline Global & 20,000 MT to $10,000,000 \mathrm{MT}$ & $400 \mathrm{~m}$ to $8 \mathrm{~km}$ \\
\hline Mass Extinction & Above $10,000,000 \mathrm{MT}$ & $>3.5 \mathrm{~km}$ \\
\hline
\end{tabular}


An interesting option when assessing the efficiency of the 5-tonne tugboat at deflecting impact threat consists on computing the total relative impact frequency of the set of impacting orbits from which a deflection above a given threshold energy is possible. This is referred here as the level of planetary protection achieved by the tugboat. In order to compute this, we must first compute the largest asteroid size that the tugboat is able to deflect from each one of the 17,518 impacting orbits represented in Figure 8. If the deflection is computed as a function of the asteroid mass, a root finding procedure can find the exact asteroid mass that allows a minimum safe deflection distance. Since the virtual impactors are defined as MOID-zero orbits, the minimum deflection to achieve a safe distance is equal to $M O I D_{\oplus}$ as in Eq.(3), here not defined as an actual MOID of the asteroid but just as a minimum distance for a particular passage. This provides the distance threshold between a collision and a near-miss. The tugboat achieved deflection distance for each simulation is computed here by using the proximal motion equations expressed as a function of the variation of the orbital elements. The variation of the orbital elements is computed by numerically integrating Gauss's planetary equations over the interval on which the tugboat is performing the deflection manoeuvre. A complete description of the models can be found at (Sanchez, Colombo, 2009).

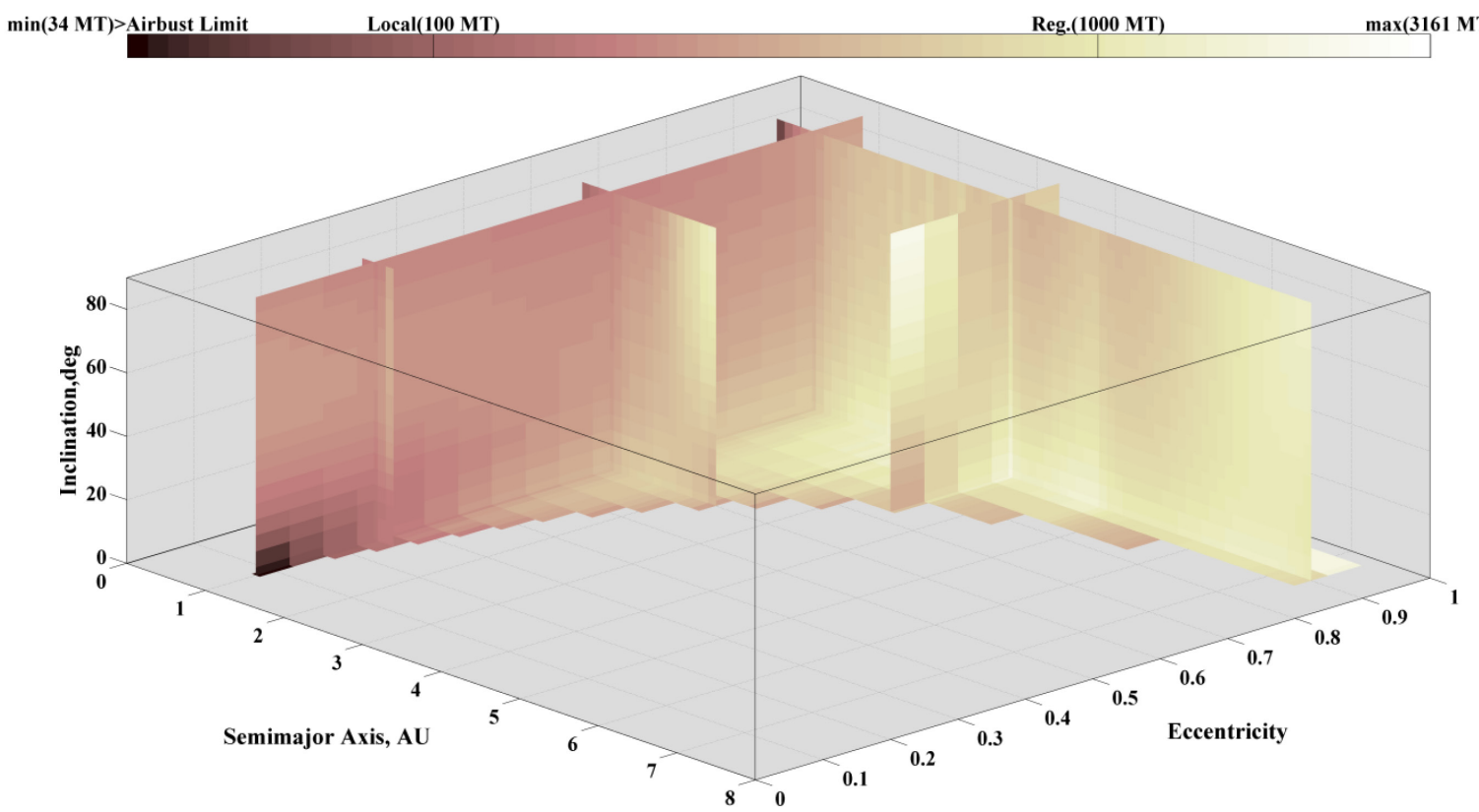

Figure 10: Deflected energy with 20 year lead time

Figure 9 shows the maximum energy deflected as a function of $\{a, e, i\}$ for the particular deflection scenario with 20 year lead time. One can see from the figure that the tugboat can provide a comprehensive protection against airburst events, since the range from 1 to $10 \mathrm{MT}$ is lower than the minimum deflected energy in Figure 9, which is 34 MT. On the other hand, any larger impact event, local or larger, is not fully protected since there are locations on the $\{a, e, i\}$ space where the maximum deflected energy does not cover completely the energy range of the event. For such cases, the efficiency of deflection of impact threats at a given $E_{\text {impact }}$ can be given by computing the total relative impact frequency of the $\{a, e, i\}$-space where the maximum deflected energy is larger than the limiting $E_{\text {impact }}$. Figure 10 shows then the levels of planetary protection achieved by the 5-tonne spacecraft for the 5 lead times analysed in this paper. The curve marked with circles summarises the data plotted in Figure 9, corresponding to a 20 years lead time. 


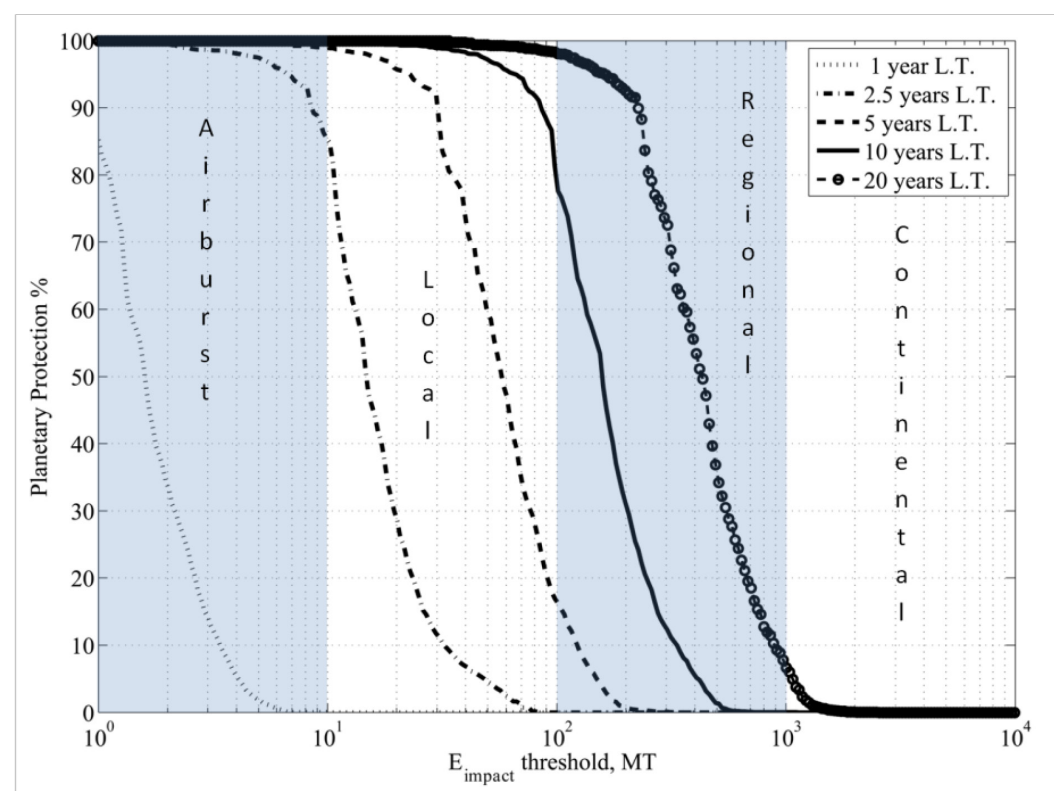

Figure 11: Planetary Protection achieved for each lead time (L.T.) analysed as a function of $E_{\text {impact }}$ threshold.

Table 2 summarizes the results shown by Figure 10 by computing only the total relative impact frequency of all the impacting orbits that can be deflected with an energy larger than the maximum impact energy of the corresponding type of impact event.

Table 2: Planetary Protection

\begin{tabular}{|r|c|c|c|c|c|}
\hline \multirow{2}{*}{ Type of Event } & \multicolumn{5}{|c|}{ Lead Time } \\
\cline { 2 - 6 } & 1 year & 2.5 years & 5 years & 10 years & 20 years \\
\hline Airburst & $0 \%$ & $85 \%$ & $99 \%$ & $100 \%$ & $100 \%$ \\
\hline Local Damage & $0 \%$ & $0 \%$ & $16 \%$ & $78 \%$ & $98 \%$ \\
\hline Regional Damage & $0 \%$ & $0 \%$ & $0 \%$ & $\sim 0 \%$ & $7 \%$ \\
\hline Continental Damage & $0 \%$ & $0 \%$ & $0 \%$ & $0 \%$ & $0 \%$ \\
\hline Global Damage & $0 \%$ & $0 \%$ & $0 \%$ & $0 \%$ & $0 \%$ \\
\hline
\end{tabular}

As the table shows, the tugboat system analysed here should be capable of protecting Earth against all airburst events if 10 years or more of lead time are available. Even with less available lead time the levels of protection at this energy level are in fact very high, except for 1 year lead time. Also, local damage events can be reasonably well protected with this deflection system if deployed on the threatening object 10 years or more before the impact. Unfortunately, the tugboat would not be able to provide sensible planetary protection against any of the three largest types. As seen in the table, shortening the lead time greatly maims the efficiency of the deflection, as to the point that for 1 year lead time a $0 \%$ protection is obtained for $10 \mathrm{MT}$ events. Nevertheless, these results demonstrate a very good capability of a relatively small tugboat system to protect the Earth against any impact threat on the low impact energy band $(<100 \mathrm{MT})$. It is important to highlight that this energy band covers the first impact-risk band, as discussed in the Introduction section (or seen at the figure 2.7 on (Shapiro et al., 2010)).

\section{CAPTURE SCENARIO}

Having shown that a small-to-medium tugboat missions has a good capability to provide planetary protection, we can now discuss the possibility of using the same tugboat system on a completely different mission: pushing a small object in order to facilitate its ballistic capture in the Earth's neighbourhood. Any envisioned future for space exploration involves both a growth in space structures and human presence in space (e.g., space solar power, space tourism or more visionary human space settlements). This, of course, implies a much larger mass of material in-use in space, for both structural mass and life support for human presence in 
space. The traditional approach to deliver material into space has always been escaping the Earth's gravity well, which is, arguably, not the most effective means, since unprocessed resources requiring less energy for transportation are in abundance in space (Sanchez and McInnes, 2011a). This paper now focuses on the possibility of moving small near Earth asteroids and inserting them onto Earth bound trajectories for later utilization. This has been previously referred as a new-moon approach to asteroid exploitation. The question that arises is then how much material could a $5000 \mathrm{~kg}$ low thrust spacecraft transport back to Earth.

The most restrictive constraint set by the tugboat, as described here, is due to its limited thrust. If the amount of thrust, and thus, acceleration of the asteroid was not a constraint, we could envisage a system able to transfer to a suitable target asteroid and provide: firstly, the small push required to give the asteroid a precise phasing with the Earth, so that the two objects meet at their MOID points; and secondly the orbital insertion necessary to place the asteroid on a bound Earth orbit. Unfortunately, the tugboat requires a long duration to provide a noticeable useful change in the velocity of the asteroid, thus allowing very limited manoeuvrability of the asteroid during Earth fly-by. This limitation entails then that the tugboat would only be able to provide small orbital changes requiring relatively long push manoeuvres and, as a consequence of this, the final Earth orbit insertion needs to be ballistic, or at least unaided by the propulsion system, perhaps with the exception of fine targeting prior to capture.

\subsection{Ballistic Capture.}

Due to its limited thrust, it is assumed that the tugboat is primarily used for the initial push which, provided long in advance of the Earth encounter, can provide the necessary phasing to meet the Earth at the point of minimum orbital distance, or MOID point. In fact, as shown by optimal asteroid deflection strategies (Vasile and Colombo, 2008), a continuous tangential thrust, which changes the phase of the threatening object with respect to the Earth without significantly changing the MOID vector, can be orders of magnitude more efficient at increasing the deflection distance than any other strategy. Similarly, then, when attempting to capture an asteroid, the slow tugboat push will be better used at changing the phase of the target asteroid orbit than at modifying the encounter geometry.

We can then assume the tugboat manipulation of the asteroid's orbit does not actually provide any noticeable change of the MOID vector, but only forces the asteroid to pass close to its own MOID point at the time that the Earth is also at its closest distance. Note that, for example, if an aero-assisted trajectory is intended, the perigee altitude must clearly be within the Earth atmosphere, and the asteroid's MOID must then allow for such a close perigee. This requires the asteroid MOID to be smaller than maximum impact distance $M O I D_{\oplus}$, as described by Eq.(3). A ballistic capture, on the other hand, does not require necessarily such a small MOID, but choosing $M O I D_{\oplus}$ as the distance threshold ensures maximum flexibility when designing the flyby maneuvre.

Figure 11 shows the expected number of objects having a MOID smaller than $M O I D_{\oplus}$ and with a hyperbolic excess velocity at Earth encounter smaller than some threshold. As seen in other studies (Kemble, 2003), Earth's ballistic escape or capture with relative velocities, $v_{\infty}$, below $1 \mathrm{~km} / \mathrm{s}$ are possible. This does not mean that all objects approaching the Earth at $1 \mathrm{~km} / \mathrm{s}$ can be ballistically captured, of course, since, in particular, the direction of the $v_{\infty}$ vector relative to the Sun is another important factor that must be adjusted for a ballistic capture trajectory to occur. Nevertheless, Figure 11 shows that there are a myriad of small objects that can be artificially phased to meet the Earth. Some of those can do so at a particularly low relative velocity, and thus, they are realistic targets for ballistic captures. Also note that the choice of maximum orbital distance in Figure 11 was left, on purpose, particularly restrictive and that the number of objects that could approach the Earth at distance smaller than the sphere of influence of the Earth and relative velocity smaller than $1 \mathrm{~km} / \mathrm{s}$ is 6 times higher than the reproduced in Figure 11 (Sanchez and McInnes, 2011b). The figure then shows that there should be a nonnegligible number of objects fulfilling ballistic capture conditions. Moreover, these potential ballistic capture targets are also small enough so that they would not pose any impact risk for Earth. It is important to stress that the objects estimated in Figure 11 are not objects expected to impact the Earth in the near future, but only objects with a minimum orbital distance (MOID) small enough that they could flyby the Earth if they were artificially forced to meet the Earth at the point of minimum orbital distance. 


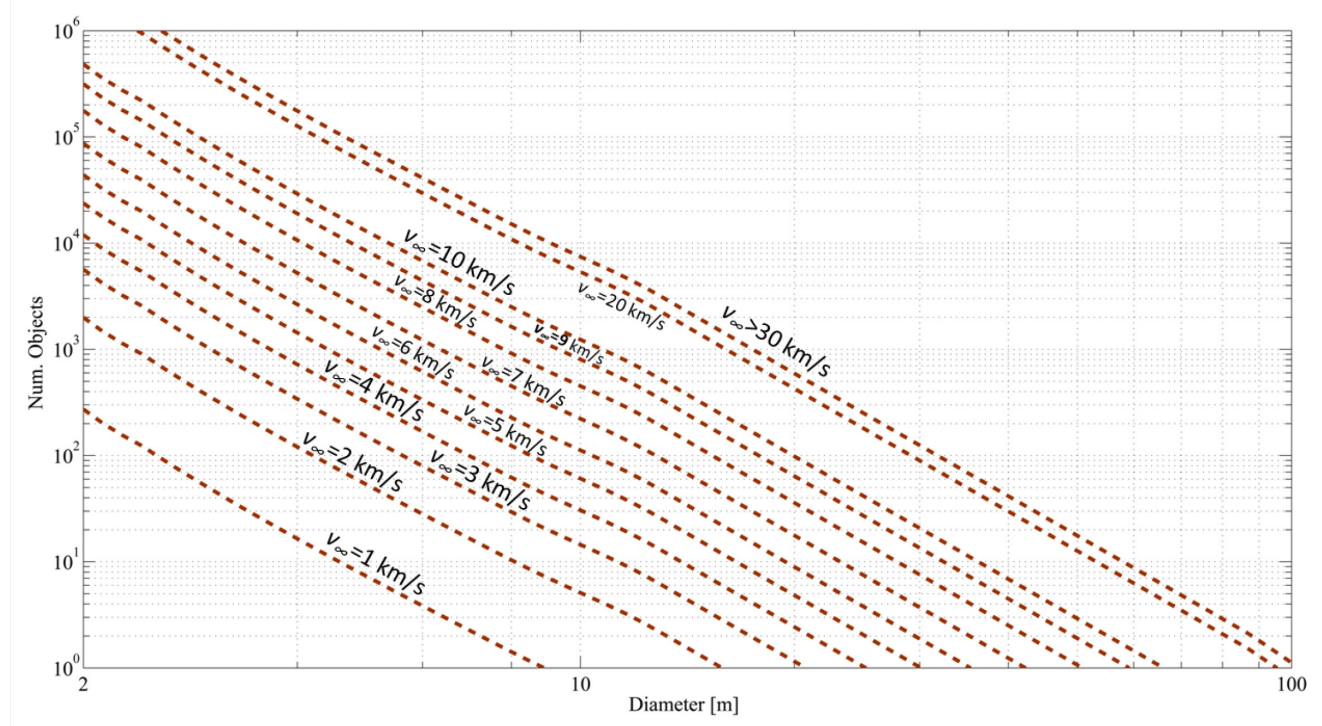

Figure 12: Total number of objects with MOID smaller than $M O I D_{\oplus}$ for a given, or lower, relative velocity at encounter. The object estimation is the average expected number of asteroids within \pm 1 meter of the size given by the abscissas axis.

The results shown in Figure 11 have been calculated by; first, defining the volume of the $\{a, e, i\}$ space that allows the hyperbolic velocity $v_{\infty}$ to be smaller than a given threshold, and second, integrating within this volume the function resulting from multiplying the population of NEO and the probability of an asteroid of having a MOID smaller than $M O I D_{\oplus}$ (Sanchez and McInnes, 2011a). The population of NEO is composed by the Bottke et al.(Bottke, Morbidelli, 2002) orbital distribution, described in section 3.1, and the latest estimates on NEO size distribution (Harris, 2007). These latest estimates seem to indicate a deviation from the commonly assumed single slope accumulative power law distribution (Stokes, Yeomans, 2003). The deviation affects the range of objects between $1 \mathrm{~km}$ and $10 \mathrm{~m}$ diameters with the maximum difference being a drop of a factor of 2/3 on the cumulative number of objects with diameter larger than $100 \mathrm{~m}$. The probability of an asteroid having a MOID smaller than a given value can be calculated similarly to Eq.(8), with the difference that Eq.(8) only accounted for two out of the 4 possible arguments of the periapsis that yield an asteroid distance to the Sun equal to the Earth's semi-major axis (a more detailed explanation can be found in (Sanchez and McInnes, 2011a)).

Note that these estimations are based on, firstly, a NEO orbital distribution independent of the asteroid size and secondly on linearised motion when computing the impact probability or MOID distance. Hence, the results should be taken only as order of magnitude estimations. Notwithstanding, it is surprising the level of accuracy when comparing frequency estimations of bolide detonation in the Earth's atmosphere using the models described here with satellite recorded frequencies (Brown et al. , 2002). The Earth is assumed to be struck with approximately 5 ktons of energy with a yearly average, as shown in (Brown, Spalding, 2002). The result computed using the models presented here is $4.2 \mathrm{ktons}$. Similarly the monthly average compares as $0.3 \mathrm{ktons}$ in (Brown, Spalding, 2002) and 0.2 ktons as computed here, while the decade average is shown to be about 50 ktons in (Brown, Spalding, 2002) and 79 ktons computed here.

\subsection{Aero-assisted Capture.}

A potential option to capture objects that have a relative velocities $v_{\infty}$ above $1 \mathrm{~km} / \mathrm{s}$ is to use grazing aeroassisted trajectories. This technique could potentially allow dissipating the excess orbital energy of the asteroid, and thus, help the Earth-bound orbital insertion to occur without the need for propulsion. This dissipation of energy is caused by the air drag experienced by the asteroid during its atmospheric passage. This drag also causes the asteroid to heat up to temperatures at which ablation of material occurs, and thus, an aero-assisted capture may reduce substantially the mass of the asteroid or alter its characteristics. Another important factor to take into account is the maximum dynamic pressure exerted on the asteroid during aero-assisted trajectories. It is generally agreed that if the maximum stagnation pressure of the air flow, $P_{\text {ram, }}$ exceeds the compressive strength 
of the asteroid $S$, then the breaking point of the object has been surpassed and the catastrophic fragmentation of the asteroid occurs (Bronshten, 1983, Hills and Goda, 1993, Svetsov et al. , 1995).

Hence, a suitable asteroid capture manoeuvre by means of grazing aero-assisted trajectories should pose the minimum possible dynamical pressure on the asteroid in order to ensure minimum mass loss while, at the same time, protecting the structural integrity of the asteroid. For the purpose of a preliminary analysis on the feasibility of the aero-assisted capture, the $\Delta v$ change achieved after a grazing atmospheric passage can be analytically approximated by assuming that the trajectory of the object is well approximated by a Keplerian orbit, together with the fact that the object would remain in the atmosphere for a very small range of true anomaly. These assumptions allow a first order approximation of the $\Delta v$ change of an aero-assisted trajectory, as (Heppenheimer, 1971):

$$
\Delta v=\left(1-e^{\mathrm{B} \rho \sqrt{2 \pi r_{p} H(e+1) / e}}\right) \cdot v_{p}
$$

where $B$ is the asteroid ballistic coefficient computed as $B=C_{D} \cdot A / 2 M$, where the drag coefficient $C_{D}$ of a sphere (e.g. 0.47) and the area-to-mass ratio $A / M$ can be also computed assuming a sphere with an average density equal to $2600 \mathrm{~g} / \mathrm{m}^{3}$ (Chesley, Chodas, 2002), $r_{p}$ is the periapsis distance and $e$ refers to the eccentricity of the fly-by orbit. The atmospheric density $\rho$ can be computed using a classic exponential model such as $\rho=\rho_{0} e^{-h / H}$, where $H$ is the scale height, $\rho_{0}$ is the sea level density and $h$ is altitude from sea level. Hence, the tugboat could trim the phasing of the asteroid in order to achieve a required periapsis distance $r_{p}$ during the Earth fly-by, and thus, achieve the required $\Delta v$ change in order to insert the asteroid into an Earth-bound orbit (i.e., a parabolic orbit, thus $v_{\infty}=0$, is assumed here to be the threshold between an Earth-bound orbit and an Earth escape orbit).

If, on the one hand, the linear momentum of the asteroid is assumed to be affected only by air drag, thus ignoring the momentum change due to the orbital motion, the momentum will then change as:

$$
m \frac{d v}{d t}=\frac{1}{2} C_{D} \rho A v^{2}
$$

where $A$ is the cross-sectional area. The mass-loss, on the other hand, can be described by:

$$
\frac{d m}{d t}=-\frac{1}{2} \frac{\kappa}{q} \rho A v^{3}
$$

where $\kappa$ is the heat-transfer coefficient, $q$ is the latent heat of vaporization and $1 / 2 \rho A v^{3}$ the kinetic energy of the oncoming stream of air. Dividing Eq.(17) by Eq.(16) and integrating, the following equation is obtained:

$$
m_{f}=m_{0} e^{\frac{\sigma}{2}\left(v_{f}^{2}-v_{0}^{2}\right)}
$$

where $\sigma$ is the ablation parameter, which is the combination of $\kappa /\left(q C_{D}\right)$. Eq.(18), although an approximation due to the fact that the Earth gravity's has been neglected, provides a good estimate of the final mass after a grazing atmospheric trajectory. In the case at hand, the grazing manoeuvre has been approximated as an impulsive change of velocity $\Delta v$ occurring at the periapsis, and thus Eq.(18) can be rewritten as:

$$
m_{f}=m_{0} e^{\frac{\sigma}{2}\left(v_{\pi}^{2}-\left(v_{\pi}+\Delta v\right)^{2}\right)}
$$

where $v_{\pi}=\sqrt{\frac{2 \mu_{\oplus}}{r_{p}}}$ and $v_{\pi}+\Delta v=\sqrt{\frac{2 \mu_{\oplus}}{r_{p}}+v_{\infty}^{2}}$.

The ablation parameter $\sigma$ in Eq.(18) plays a paramount role when estimating the mass loss of the asteroid. This parameter, although generally assumed to be a constant along the atmospheric trajectory of the asteroid (Bronshten, 1983, Hills and Goda, 1993), is found to vary with altitude, velocity, mass and size of observed meteoroids. However, if the ablation process is dominated by a short interval of the trajectory a constant value 
of $\sigma$ can be taken as the effective ablation parameter of the grazing trajectory. In this paper an effective $\sigma$ equal to $2.1 \times 10^{-8} \mathrm{~s}^{2} / \mathrm{m}^{2}$ is taken as a reasonable effective value (Bronshten, 1983). Nevertheless it is expected that large meteoroids ( $>1 \mathrm{~m}$ ) would actually suffer lower levels of ablation due to the strong shielding caused by the screening effect of vaporizing material (Svetsov, Nemtchinov, 1995). The ablation parameter $\sigma$ could well be smaller than $5 \times 10^{-10} \mathrm{~s}^{2} / \mathrm{m}^{2}$, if considering other studies (Cook et al. , 1963) and other theoretical models (Svetsov, Nemtchinov, 1995), which would represent a mass loss several times lower than that calculated using $2.1 \times 10^{-8} \mathrm{~s}^{2} / \mathrm{m}^{2}$. For large asteroids however a "mechanical ablation" process may also take place, in which small fragments of material can be blown off instead of being ablated. Thus, $2.1 \times 10^{-8} \mathrm{~s}^{2} / \mathrm{m}^{2}$ is considered here a cautious estimate of the ablation parameter $\sigma$, allowing conservative estimates of the mass loss.

As already mentioned, the structural integrity of the asteroid also needs to be ensured. The break-up limit is reached when the stagnation pressure of the air flow $P_{\text {ram }}$ equals the compressive strength of the material $S$ :

$$
P_{r a m} \equiv \rho \cdot v^{2}=S \text {. }
$$

The $P_{\text {ram }}$ maximum would occur at the periapsis of the asteroid orbit, where both air density and velocity are maximum. Hence, it will be considered here that a grazing aero-assisted capture is not feasible if $P_{\text {ram }}$ at the periapsis exceeds the material compressive strength:

$$
\rho\left(r_{p}\right) \cdot v_{p}^{2}=\rho\left(r_{p}\right) \cdot\left(v_{\pi}+\Delta v\right)^{2}<S .
$$

The periapsis velocity $v_{p}$ is assumed to be the periapsis velocity of the asteroid prior to the grazing atmospheric manoeuvre (i.e., $v_{\pi}+\Delta v$ ), which defines a maximum threshold that cannot be exceeded. Eq.(21) defines then the maximum $\Delta v$ manoeuvre that can be allowed for an asteroid of material strength $S$. Eq.(15), on the other hand, defines the altitude of periapsis $r_{\mathrm{p}}$ required to achieve a given $\Delta v$. Equations (15) and (21) require to be solved together.

Figure 12 shows the expected material available with orbits such that could potentially be aero-captured by setting a maximum ram pressure not exceeding a given asteroid material strength. Asteroid material strengths are taken from Hills and Goda (Hills and Goda, 1993), where dustball (e.g., cometary body or rubble pile asteroid) is assumed to have a strength approximately $10^{6} \mathrm{~N} / \mathrm{m}^{2}$, stony (chondritic) asteroids approximately $10^{7}$ $\mathrm{N} / \mathrm{m}^{2}$ and nickel-iron asteroids approximately $10^{8} \mathrm{~N} / \mathrm{m}^{2}$. The atmospheric model used in Figure 12 is taken from Vallado (Table 8.4) (Vallado, 2007), although a simpler model such as that used in (Hills and Goda, 1993) yields results similar to the ones represented here. The accuracy of these models is considered to be sufficient for a preliminary analysis on the feasibility of the aero-assisted capture. It is assumed that while longitudinal and latitudinal atmospheric variations can be predicted and therefore accounted for, any other source of unpredictable error, such as changes on solar flux, could be compensated by adding additional margins on the maximum ram pressure allowed. Nevertheless, uncertainties on material properties of asteroids (i.e., asteroid material strength) and interaction with the atmosphere (i.e., ablation parameter) would have a higher effect on the manoeuvre outcome, than atmospheric variations. 


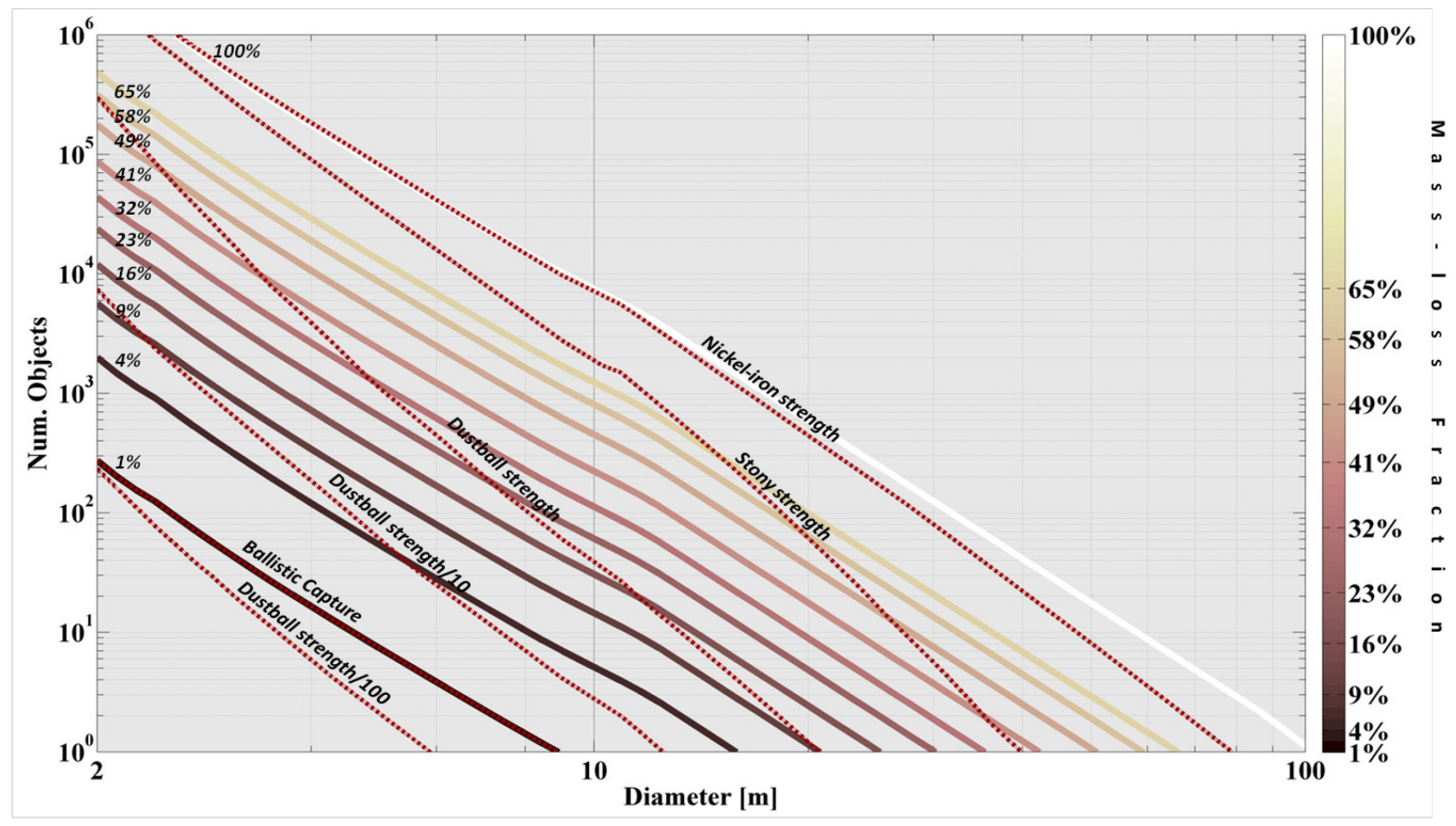

Figure 13: Number of capturable objects. The object estimation is the statistical expected number of asteroids within \pm 1 meter of the size given by the abscissas axis. The coloured legend represent the mass loss fraction due to the atmospheric passage.

Figure 12 also represents the mass loss fraction computed for objects approaching the Earth with the same excess velocities used in Figure 11. The information in the figure reads as follow: let us assume that we want to know the number of $10 \mathrm{~m}$ diameter objects that can be captured by the low thrust tugboat method proposed here. Figure 12 shows that statistically there should be less than 1 object of this size that could be captured ballistically. By allowing maximum ram pressures of $10^{5} \mathrm{~N} / \mathrm{m}^{2}(10$ times lower than the dustball limiting strength) close to 3 objects should be found. By allowing $10^{6} \mathrm{~N} / \mathrm{m}^{2}$ (i.e., dustball limit strength) close to 40 objects should be found. Note from the coloured ablation isolines that the maximum level of ablation of those objects is lower than $23 \%$. This does not mean that all 40 objects are expected to have a $23 \%$ mass loss during the atmospheric capture, but only in the worst case. 5 objects, for example will have a level of ablation lower than $4 \%$. We can also allow for higher ram pressures, i.e., $10^{7} \mathrm{~N} / \mathrm{m}^{2}$ and $10^{8} \mathrm{~N} / \mathrm{m}^{2}$, and the number of available objects increases enormously. The mass loss fraction however also increases to the point that nickel-iron object may ablate completely.

Apart from the obvious problem that entails the asteroid's mass loss with grazing aero-assisted manoeuvres, the two higher levels of material strength need to be considered cautiously. Some analysis on fireball data seem to indicate that most of the Earth impacting small objects have a fragmentation limit only slightly higher than the $10^{6} \mathrm{~N} / \mathrm{m}^{2}$ (Svetsov, Nemtchinov, 1995). Finally, objects that can be potentially captured with aero-assisted trajectories yielding maximum ram pressures around $10^{4} \mathrm{~N} / \mathrm{m}^{2}$ could also be captured ballistically, thus avoiding any atmospheric contact.

\subsection{Object Phasing Maneuvre.}

Figures 11 and 12 have given some estimates on the amount material available that could be gravitationally captured at Earth, but it has yet to be assessed how much of this material could actually be pushed by the tugboat to meet the Earth at the encounter point, and thus proceed with the capture maneuvre. As noted in Section 3.1, the mean anomaly of asteroids can be modelled as a uniform random variable. Thus, if the range of mean anomaly from where the tugboat has the capability to phase an asteroid is computed, then the probability that an asteroid could actually be phased, and therefore captured, is simply the fraction between the size of this range and $2 \pi$. Figure 14 depicts the phasing procedure. The paper assumes the tugboat reaching the asteroid at a time $t_{i}$ and performing the pushing maneuvre during the length of time $\left(t_{f}-t_{i}\right)$. In order to assess the capability of the tugboat to provide phase changes to the asteroid orbit, we will only consider secular changes. Thus, the 
tugboat phasing maneuvre should correct the difference in mean anomaly $M$ that would allow the asteroid to meet the Earth at the MOID point. This can be expressed as:

$$
\Delta M=\left[n_{f}\left(t_{M O I D}-t_{f}\right)+\int_{t_{i}}^{t_{f}} n \cdot d t\right]-n_{i}\left(t_{\text {MOID }}-t_{i}\right)
$$

where $n$ is the asteroid mean motion and the subscripts $i, f$ and MOID refer respectively to the arrival time, the time at the end of the pushing manoeuvre and the impact time.

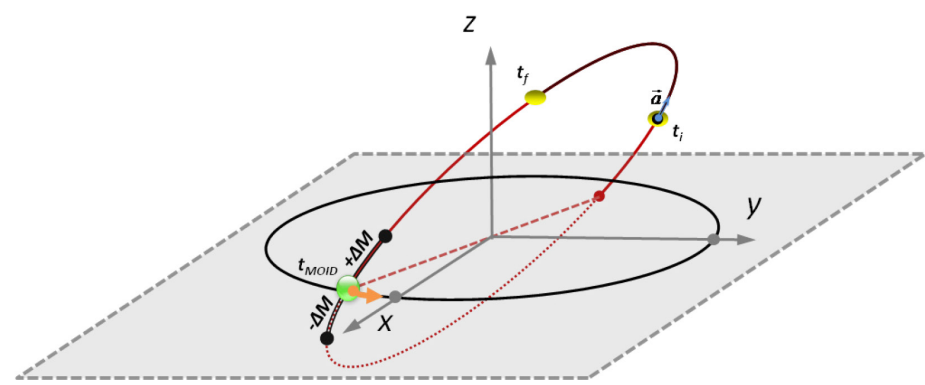

Figure 14: Schematic of the asteroid phasing manoeuvre.

The expression within the brackets in the right side of Eq. (22) represents the mean anomaly of the perturbed asteroid, thus between the time $t_{f}$ and $t_{M O I D}$ the asteroid coasts without further change in its mean anomaly. The time $t_{M O I D}$ is the time at which the encounter is intended, when both the Earth and asteroid should be at their MOID points. On the other hand, the expression $n_{i}\left(t_{M O I D^{-}} t_{i}\right)$ yields the mean anomaly of the unperturbed asteroid, and thus, the difference between the two is the achieved change of phase $\Delta M$. The time spanned between $\left(t_{M O I D}{ }_{i}\right)$ was defined in Section 2.1 as the lead time. We will analyse here the phasing capability of the tugboat by using the same lead times as in Section 3 (i.e., 1, 2.5, 5, 10 and 20 years). Note that when the tugboat is set to phase an asteroid with a lead time of, for example, 20 years, it may not require the full 20 years. In fact, since the Earth will be at the same position every year, an asteroid with a random mean anomaly may find better opportunities to be phased at a time $t_{M O I D}^{m}=t_{M O I D}-m \cdot P_{\oplus}$, where $m$ is a positive integer smaller than the lead time (in years) and $P_{\oplus}$ is the period of the Earth.

From Eq.(22), we can re-write it as:

$$
\Delta M^{m}=\left[n\left(t_{M O I D}^{m}-t\right)\right]_{t_{i}}^{t_{f}}+\int_{t_{i}}^{t_{f}} n \cdot d t
$$

where $\Delta M^{\mathrm{m}}$ refers to the change of mean anomaly achieved at the encounter occurring at $t_{M O I D}^{m}$. Note also that the length of the pushing manoeuvre $\left(t_{f}-t_{i}\right)$ needs to be set in order to achieve the maximum change in mean anomaly. The same heuristic model as in Section 3 is used here to assess the duration of the pushing manoeuvre $\left(t_{f}-t_{i}\right)$, which is taken from ref. (Sanchez, Colombo, 2009). The expression in Eq.(23) can be modified by recalling the integral by parts transformation into:

$$
\Delta M^{m}=\int_{t_{i}}^{t_{f}}\left(t_{M O I D}^{m}-t\right) \frac{d n}{d t} \cdot d t
$$

and using the chain rule of derivatives, we see that

$$
\frac{d n}{d t}=\frac{d n}{d a} \cdot \frac{d a}{d t}=\frac{-3}{2} \sqrt{\frac{\mu_{S}}{a^{5}}} \cdot \frac{d a}{d t} .
$$

Finally, by using the Gauss planetary equations (Battin, 1999), we can write the time derivative of the semimajor axis as: 


$$
\frac{d a}{d t}=\frac{2 a^{2} v}{\mu_{S}} a_{t},
$$

where $a_{t}$ is the tangential component of the tugboat acceleration and $v$ is the orbital velocity. Hence, Eq.(25) results in:

$$
\frac{d n}{d t}=\frac{-3}{\sqrt{\mu_{S} a}} \cdot v \cdot a_{t}
$$

The integration at Eq.(24) can be now written as:

$$
\Delta M^{m}=\frac{-3}{\sqrt{\mu_{S}}} \int_{t_{i}}^{t_{f}}\left(t_{M O I D}^{m}-t\right) \frac{v \cdot a_{t}}{\sqrt{a}} \cdot d t .
$$

A very similar formulation can be found in previous work on asteroid deflection (Colombo et al. , 2009, Izzo, 2007).

In this case, the acceleration that the tugboat provides is extremely small and, thus, the change in semi-major axis will also be small. On the other hand, the acceleration provided $a_{t}$ remains almost constant during the whole push maneuvre, since the mass of the propellant consumed is only a tiny fraction of the combined mass of the asteroid and spacecraft. Considering this, the following expression is then a good approximation of the integral in Eq.(28):

$$
\Delta M^{m}=\frac{-3 a_{t}}{\sqrt{\mu_{S} a}} \int_{t_{i}}^{t_{f}}\left(t_{M O I D}^{m}-t\right) v(t) \cdot d t,
$$

where $v(t)$ is the orbital velocity of the asteroid and can be computed by assuming Keplerian motion. The integral in Eq.(29) can be integrated numerically and provides a good and quick estimation of the phasing capability of the tugboat at each encounter opportunity. Finally, each set of $M_{M O I D}^{m} \pm \Delta M^{m}$, where $M_{M O I D}^{m}$ is the mean anomaly necessary for the asteroid to meet the Earth at the MOID point at the $m$ encounter, is mapped to the same epoch (e.g., $t_{i}$ ). The latter is done in order to compute the total range of feasible mean anomalies, where feasible refers here to mean anomalies that allow the tugboat to artificially force an encounter of the asteroid with the Earth. Note that a range of feasible mean anomalies $M_{M O I D}^{m} \pm \Delta M^{m}$ at time $t_{M O I D}^{m}$ may be overlapping with another set and not apparently do so simply because each set is expressed at a different time or epoch. Finally, and as previously described, the probability that an asteroid could be artificially phased and forced to meet the Earth is yielded by the fraction between the total feasible range of mean anomalies and $2 \pi$.

Let us now consider an example of the relative ease with which the 5,000 kg tugboat system could push a 10 -meter object to an encounter with Earth. Figure 12 showed an estimate of several tens of $10 \mathrm{~m}$ objects with the potential to be captured at Earth with aero-assisted trajectories posing a maximum ram pressure threshold of $10^{6} \mathrm{~N} / \mathrm{m}^{2}$. In order to avoid stagnation pressures higher than this limit, these objects would have to approach the Earth with a maximum excess velocity of approximately $4.31 \mathrm{~km} / \mathrm{s}$. The latter requirement defines a Keplerian sub-space volume as shown in the right-top corner of Figure 14 and, if the orbital distribution of objects with $M O I D \leq M O I D_{\oplus}$ is integrated within this volume, the total probability of finding such an object is $1.5 \times 10^{-6}$. Assuming Harris' population estimates (Harris, 2007), which envisages a total of 25 million objects with diameters ranging from 9 to 11 meters, the number of existing objects with the required orbital elements should be an average of 37. The mean anomaly of these objects is not known, but it is known that its probability density is well approximated by a uniform distribution. By using the procedure described in this section the probability of each of these objects having a mean anomaly such that the tugboat could phase them with the Earth can be computed as a function of lead time (see Figure 14). Note that the integration in Eq.(29) is not only a function of $\{a, e, i\}$, but also a function of the asteroid diameter and lead time. The tangential acceleration $a_{t}$, in Eq.(29), is the result of the ratio $M_{A s t} / T_{\text {eff }}$ where $M_{A s t}$ is the asteroid mass, which is assumed here to be only a function of diameter, and $T_{\text {eff }}$ is the effective thrust provided by the tugboat. The effective thrust $T_{\text {eff }}$ is computed as described in Section 2. Finally, Figure 14 shows that the larger the lead time the larger the number of objects 
that can be pushed onto an encounter with the Earth. This was clearly expected, since an early implementation of the phasing manoeuvre grants a more efficient phasing.

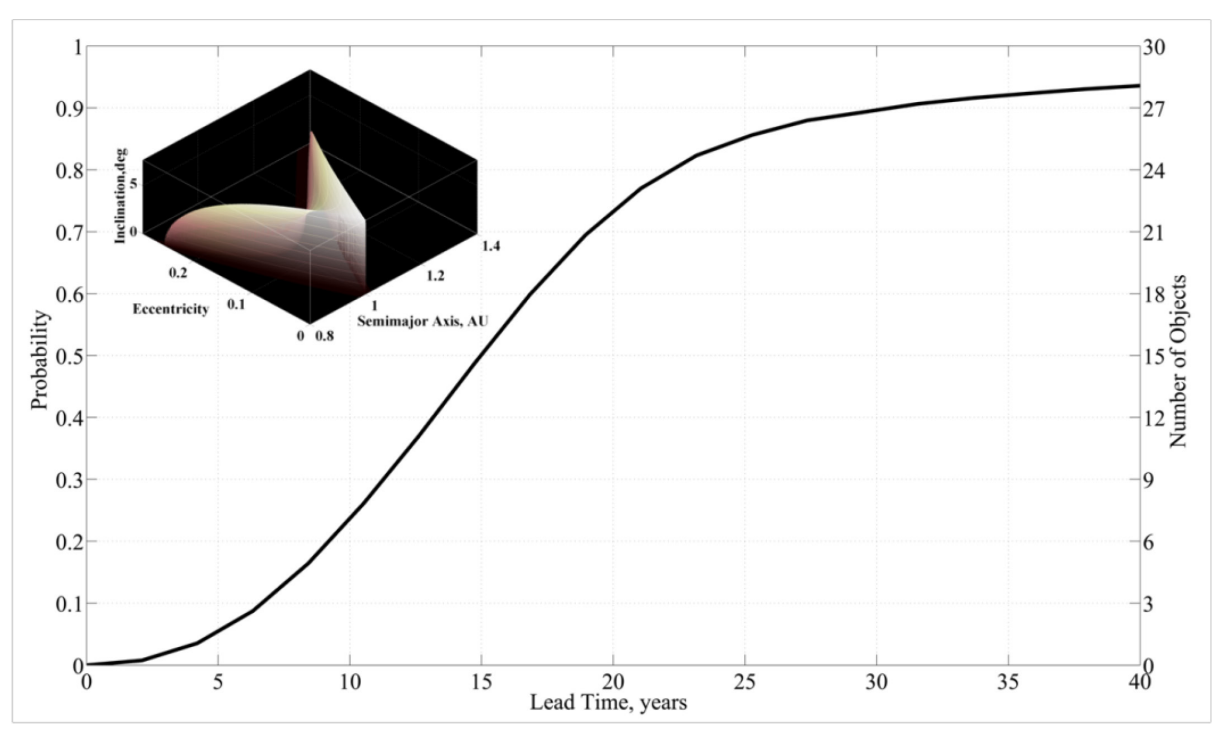

Figure 15: Average probability that a 10-m asteroid on an orbit such that $v_{\infty}<4.32 \mathrm{~km} / \mathrm{s}$ and $M O I D \leq M O I D_{\oplus}$ could be phased with the Earth by the 5-tonne tugboat system.

Finally, Figure 11 can now be reproduced showing the number of objects that can effectively be captured by the tugboat system described in this paper. Figure 15 shows four different capturing methodologies: A) a completely ballistic capture, B) a capture posing maximum stagnation pressures of $10^{5} \mathrm{~N} / \mathrm{m}^{2}$ (dustball $/ 10$ ), C) a capture posing maximum stagnation pressures of $10^{6} \mathrm{~N} / \mathrm{m}^{2}$ (dustball), D) a capture posing maximum stagnation pressures of $10^{7} \mathrm{~N} / \mathrm{m}^{2}$ (stony) and maximum allowed level of ablation of $50 \%$. The figure shows the total number of objects, represented by a thicker line, and the fraction of them that can be phased within a fixed lead time. The latter is represented by a set of thinner lines marked with different symbols. 

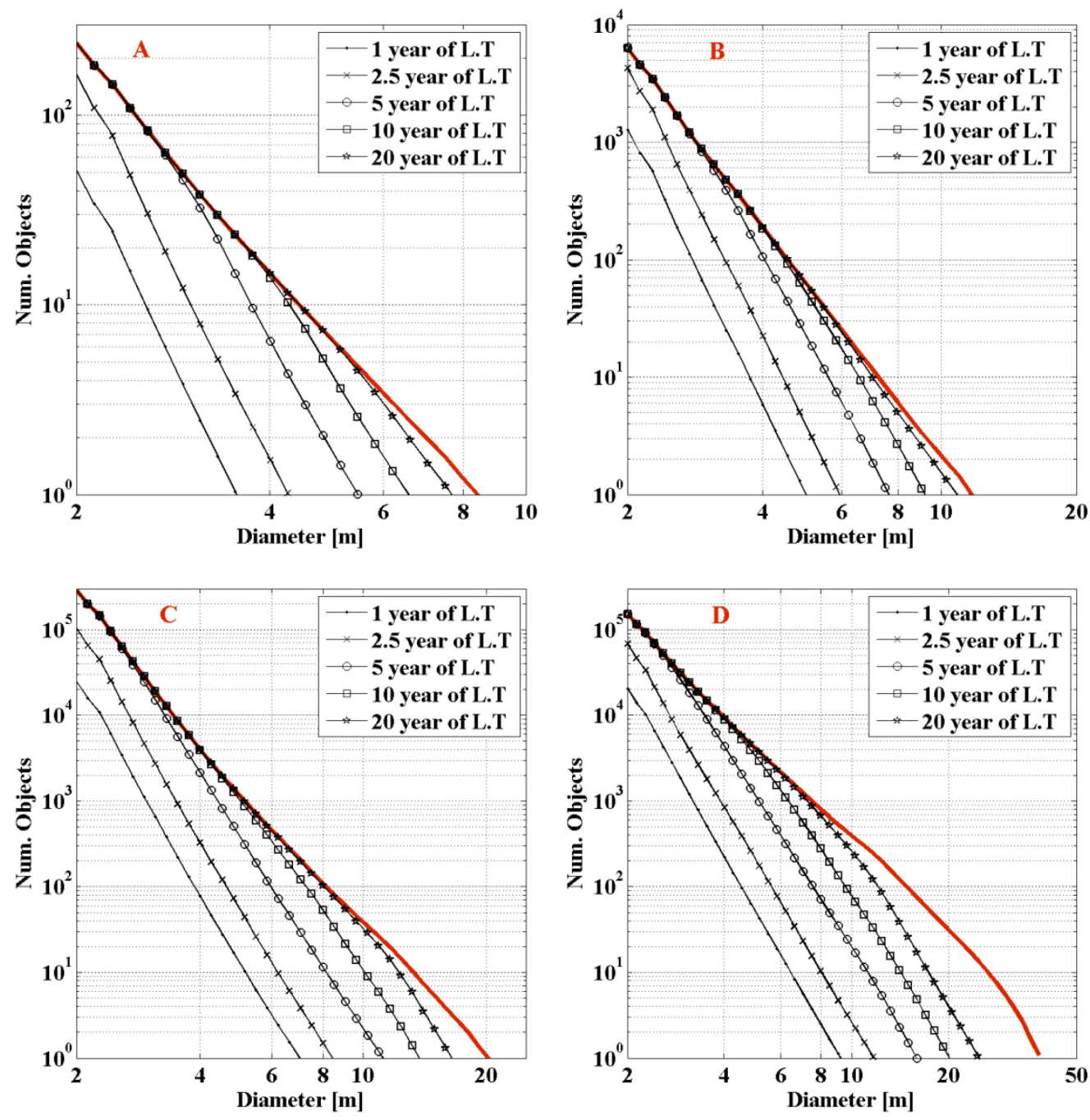

Figure 16: Total number of statistical available objects assuming a 1, 2.5, 5, 20 and $\infty$ year lead time for the 5-tonne tugboat to schedule a phasing manoeuvre. The object estimation is the statistical expected number of asteroids within \pm 1 meter of the size given by the abscissas axis.

As shown in Figure 15A, if the tugboat is deployed at the asteroid with 1 year of lead time, the largest object that can be transported to the Earth's neighbourhood and ballistically captured is 3.5 meters diameter. Assuming a spherical shape and an average density, this represents 60 tonnes of asteroid material and a mass return of 12 times the launch mass. Of course, this leverage increases rapidly if either aero-assisted trajectories or longer lead times are used. If aero-assisted capture is used, the largest capturable object depends on both the level of dynamical pressure and mass loss that is allowed during the aero-capture manoeuvre. In the case that an objects is known to be a stony or metallic asteroid, and thus believed to withstand dynamical pressures at least of $10^{7}$ $\mathrm{N} / \mathrm{m}^{2}$ and a maximum allowed mass loss of $50 \%$ is permitted (Figure 15C), an object up to 9 meters diameter could be captured with 1 year of lead time, or a $25-\mathrm{m}$ object if the asteroid is shepherded long in advance (20 year lead time). Table 3 summarizes the mass of the largest single asteroid that the tugboat could potentially capture, and between parentheses the mass fraction returned compared with the initial wet mass of the tugboat. 
Table 3: Largest mass returned to Earth - parenthesis: fraction of returned mass compared with the initial wet mass of the spacecraft.

\begin{tabular}{|c|c|c|c|c|c|}
\hline \multirow[t]{2}{*}{ Type of Capture } & & \multicolumn{3}{|c|}{ Lead Time } & \\
\hline & 1 year & 2.5 years & 5 Years & 10 Years & 20 years \\
\hline Ballistic & $>60 t(12)$ & $>105 t(21)$ & $>220 t(44)$ & $>385 t(77)$ & $>615 t(123)$ \\
\hline $\begin{array}{l}\text { Dustball Str. } / 10 \\
\left(P_{\mathrm{ram}}<10^{5} \mathrm{~N} / \mathrm{m}^{2}\right)\end{array}$ & $>170 t(34)$ & $>285 t(57)$ & $>615 t(123)$ & $>1,070 t(214)$ & $>1,675 t(348)$ \\
\hline $\begin{array}{r}\text { Dustball Strength } \\
\left(P_{\mathrm{ram}}<10^{6} \mathrm{~N} / \mathrm{m}^{2}\right)\end{array}$ & $>470 t(94)$ & $>815 t(163)$ & $>1,935 \mathrm{t}(387)$ & $>3,570 t(714)$ & $>6,175 \mathrm{t}(1235)$ \\
\hline $\begin{array}{r}\text { Stony Strength } \\
\left(P_{\text {ram }}<10^{7} \mathrm{~N} / \mathrm{m}^{2}\right) \\
\text { and } 50 \% \text { max } \\
\text { mass-loss }\end{array}$ & $>1,080 t(216)$ & $>2,190 t(438)$ & $>5,490 \mathrm{t}(1098)$ & $>11,185 \mathrm{t}(2237)$ & $>21,215 t(4243)$ \\
\hline
\end{tabular}

Note that the leveraged mass returns described in Table 3 are only for the single largest object and not for every mission. Once the largest object has been captured, the remaining objects will be smaller, following the size population as described by Figure 15. Another issue to note: after the aero-assisted manoeuvre has been performed successfully, the perigee will require to be raised. Multiple grazing aero-assisted passages may be possible for some particular configurations of the Sun direction with respect the asteroid's line of apse. This possibility can be envisaged to be used to reduce the eccentricity of the orbit. In general, however, significant pericentre variation is likely to occur due to solar and lunar perturbations, and thus the evolution of the postcapture orbit should be carefully analysed in order to ensure long term stability. This analysis should be attempted in future work on the possibility of asteroid capture. We can yet envisage that the asteroid's orbit will always require to be stabilized above the Earth's atmosphere. For this, a system capable of providing a higher thrust may be required (e.g., chemical propulsion or a kinetic impactor), or alternatively, solar and lunar gravity perturbation could also be used advantageously. Nevertheless, the work presented here shows that possible synergies between planetary protection and asteroid exploitation do exist for space systems capable of altering the linear momentum of an asteroid, or comet. The results on Table 3 can also be envisaged as a limit threshold for science sample return missions.

\section{CONCLUSIONS}

This paper has demonstrated the capability of a 5-tonne low thrust tugboat system to deflect potential impact threats, but also to transport useful quantities of asteroid material to a bound Earth orbit. Such a system would require levels of investment comparable to medium-to-large current science exploration missions. It would offer complete protection against impact threats with energies lower than $10 \mathrm{MT}$, if the tugboat could be deployed at the threatening object 10 years or more before the impact (i.e., lead time longer than 10 years). With the same early deployment, the system would also offer very reasonable levels of protection against $100 \mathrm{MT}$ impacts. In addition, high levels of protection are provided for 10 MT events (i.e., Airburst type like Tunguska) with shorter deployment times. These levels of protection, of course, assume that the threatening objects are discovered in advance, allowing the tugboat to be launched and deployed within the required lead time.

The same low-thrust tugboat system has been envisaged here as a potential system to transport small asteroids for later utilisation in Earth orbit. Objects ranging from 3.5 to 25 meters diameter could be inserted into Earth-bound orbits with the support of a 5-tonne tugboat system. The tugboat has the capacity to trim the orbit of the asteroid enough to ensure that the Earth and the capturable target object meet at their common MOID point. The size of the largest capturable object depends then on the assumed available lead time and the technique used for capture; either ballistic or aero-assisted. The latter requires to be designed for the particular material strength of target object, including the safety margins. However, even the most conservative case analysed, 1 year lead time and completely ballistic capture, a returned mass of 60 tonnes is shown to be possible. This represents a very significant leverage in mass delivered to Earth escape. Most importantly, such small objects would not pose any impact hazard to Earth, in case of a failed manoeuvre. The results shown here, of course, are only statistical averages. Thus, even if no objects are known with orbits such that could they be 
easily captured, the analysis presented here shows that there is a significant probability that objects of similar size exist.

\section{ACKNOWLEDGEMENTS}

We thank William Bottke for kindly providing us with the NEA distribution data. We also thank the Faculty of Engineering and ICSS High Performance Computer, University of Strathclyde. The work reported was supported by European Research Council grant 227571 (VISIONSPACE). We thank the anonymous reviewers for their helpful comments.

\section{REFERENCES}

Alvarez, L.W., Alvarez, W., Asaro, F., Michel, H.V. Extraterrestrial cause for the cretaceous-tertiary extinction. Science 208, 1095-108, 1980.

Battin, R.H. Introduction to the Mathematics and Methods of Astrodynamics. American Institute of Aeronautics and Astronautics, Reston, Virginia, 1999.

Betts, J.T. Survey of numerical methods for trajectory optimization. J. Guid. Control 21, 193-207, 1998.

Bottke, W.F., Morbidelli, A., Jedicke, R., et al. Debiased orbital and absolute magnitude distribution of the nearearth objects. Icarus 156, 399-433, 2002.

Bronshten, V.A. Physics of Meteoric Phenomena. D. Reidel Publishing Co., Dordrecht, 1983.

Brown, P., Spalding, R.E., ReVelle, D.O., Ragliaferri, E., Worden, S.P. The flux of small near-earth objects colliding with the earth. Nature 420, 294-6, 2002.

Chesley, S.R., Chodas, P.W., Milani, A., Yeomans, D.K. Quantifying the risk posed by potential earth impacts. Icarus 159, 423-32, 2002.

Colombo, C., Vasile, M., Radice, G. Semi-analytical solution for optimal low-thrust deflection of near-earth objects. J. Guid. Control 32, 796-809, 2009.

Cook, A.F., Jacchia, L.G., McCrosky, R.E. Luminous efficiency of iron and stone asteroidal meteors. SM. C. Astro. 7, 209-20, 1963.

De Pascale, P., Vasile, M. Preliminary design of low-thrust multiple gravity assist trajectories. J. Spacecraft Rockets 43, 1065-76, 2006.

Edward, T.L., Stanley, G.L. Gravitational tractor for towing asteroids. Nature 438, 177-8, 2005.

Harris, A.W. An update of the population of neas and impact risk. B. Am. Astron. Soc. 39, 511, 2007.

Heppenheimer, T.A. Approximate analytic modeling of a ballistic aerobraking planetary capture. J. Spacecraft Rockets 8, 554-5, 1971.

Hills, J.G., Goda, M.P. The fragmentation of small asteroids in the atmosphere. Astron. J. 105, 1114-44, 1993.

Ivashkin, V.V., Smirnov, V.V. An analysis of some methods of asteroid hazard mitigation for the earth. Planet. Space Sci. 43, 821-5, 1995.

Izzo, D. Optimization of interplanetary trajectories for impulsive and continuous asteroid deflection. J. Guid. Control 30, 401-8, 2007.

James, R.W., Wiley, J.L. Space Mission Analysis and Design. Microcosm Press, El Segundo, California, 2003. Kemble, S. Interplanetary missions utilising capture and escape through the lagrange points. In: Federation, I.A., (Ed.). 54th International Astronautical Congress. Bremen, Germany, 2003.

Kleiman, L.A. Project icarus: An MIT Student Project in Systems Engineering. The MIT Press, Cambridge, Massachusetts, 1968.

Langmaier, J., Elliott, J., Clark, K., Pappalardo, R., Reh, K., Spilker, T. Assessment of alternative europa mission architectures. JPL Publication 08-01. NASA, 2008.

Morrison, D. The spaceguard survey: Report of the nasa international near-earth-object detection workshop. Morrison, David ed. National Aeronautics and Space Administration, Washington, D.C., 1992.

Opik, E.J. Collision probabilities with the planets and the distribution of interplanetary matter. P. Roy. Irish Acad. 54, 165-99 1951.

PA\&E. 2006 Near Earth Object Survey and Deflection Study: Final report (draft). NASA's Office of Program Analysis and Evaluation, 2007.

Rayman, M.D., Fraschetti, T.C., Raymond, C.A., Russell, C.T. Dawn: A mission in development for exploration of main belt asteroids vesta and ceres. Acta Astronaut. 58, 605-16, 2006.

Sanchez, J.P. Asteroid Hazard Mitigation: Deflection Models and Mission Analysis. University of Glasgow, Glasgow, 2009.

Sanchez, J.P., Colombo, C., Vasile, M., Radice, G. Multi-criteria comparison among several mitigation strategies for dangerous near earth objects. J. Guid. Control 32, 121-42, 2009. 
Sanchez, J.P., McInnes, C.R. Asteroid resource map for near-earth space. J. Spacecraft Rockets 48, 153-65, 2011a.

Sanchez, J.P., McInnes, C.R. On the ballistic capture of asteroids for resource utilization. 62nd International Astronautical Congress. IAF, Cape Town, SA, $2011 \mathrm{~b}$.

Scheeres, D.J., Schweickart, R.L. The mechanics of moving asteroids. Planetary Defense Conference. AIAA 2004-1446 ed. American Institute of Aeronautics and Astronautics, Orange County, California, 2004.

Shapiro, I. I., A'Hearn, M., Vilas, F., et. al. Defending Planet Earth: Near-Earth Object Surveys and Hazard Mitigation Strategies. National Research Council, Washington, D.C., pp. 153, 2010.

Steel, D. Tunguska at 100. Nature 453, 1157-9, 2008.

Stokes, G.H., Yeomans, D.K., Bottke, W.F., et al. Study to Determine the Feasibility of Extending the Search for Near-Earth Objects to Smaller Limiting Diameters. In: Near-Earth Object Science Definition, T., (Ed.). NASA, Report NASA report 030825, 2003.

Svetsov, V.V., Nemtchinov, I.V., Teterev, A.V. Disintegration of large meteoroids in earth's atmosphere: Theoretical models. Icarus 116, 131-53, 1995.

Vallado, D.A. Fundamentals of Astrodynamics and Applications. Third Edition ed. Microcosm Press and Springer, Hawthorne Press, 2007.

Vasile, M., Colombo, C. Optimal impact strategies for asteroid deflection. J. Guid. Control 31, 858-72, 2008.

Vasile, M., Maddock, C. On the deflection of asteroids with mirrors. Celest. Mech. Dyn. Astr. 107, 265-84, 2010. 